\title{
Untimely TGF $\beta$ responses in COVID-19 limit antiviral functions of NK cells
}

https://doi.org/10.1038/s41586-021-04142-6

Received: 30 March 2021

Accepted: 14 October 2021

Published online: 25 October 2021

Check for updates

\author{
Mario Witkowski ${ }^{1,2,3,31 凶}$, Caroline Tizian ${ }^{1,2,31}$, Marta Ferreira-Gomes ${ }^{4,31}$, Daniela Niemeyer ${ }^{5,6}$, \\ Terry C. Jones ${ }^{5,6,7}$, Frederik Heinrich ${ }^{4}$, Stefan Frischbutter ${ }^{8,9}$, Stefan Angermair ${ }^{10}$, \\ Thordis Hohnstein ${ }^{1,2}$, Irene Mattiola, ${ }^{1,2}$, Philipp Nawrath ${ }^{1,2}$, Sophie McEwen ${ }^{1,2}$, Silvia Zocche ${ }^{11}$, \\ Edoardo Viviano $^{12}$, Gitta Anne Heinz ${ }^{4}$, Marcus Maurer ${ }^{8,9}$, Uwe Kölsch $^{13}$, Robert Lorenz Chua ${ }^{14}$, \\ Tom Aschman ${ }^{15}$, Christian Meisel ${ }^{13,16}$, Josefine Radke ${ }^{15}$, Birgit Sawitzki ${ }^{16}$, Jobst Roehmel ${ }^{17}$, \\ Kristina Allers $^{18}$, Verena Moos ${ }^{18}$, Thomas Schneider ${ }^{18}$, Leif Hanitsch ${ }^{16}$, Marcus A. Mall ${ }^{17,19}$, \\ Christian Conrad ${ }^{14}$, Helena Radbruch ${ }^{15}$, Claudia U. Duerr ${ }^{20}$, Joseph A. Trapani ${ }^{21}$, \\ Emanuela Marcenaro ${ }^{22}$, Tilmann Kallinich ${ }^{17,23}$, Victor M. Corman ${ }^{5,6}$, Florian Kurth ${ }^{24,25}$, \\ Leif Erik Sander ${ }^{24}$, Christian Drosten $^{5,6}$, Sascha Treskatsch ${ }^{10}$, Pawel Durek $^{4}$, \\ Andrey Kruglov ${ }^{26,27,28}$, Andreas Radbruch ${ }^{29}$, Mir-Farzin Mashreghi ${ }^{4,30,32}$ \& \\ Andreas Diefenbach ${ }^{1,2,3,32} \bowtie$
}

SARS-CoV-2 is a single-stranded RNA virus that causes COVID-19. Given its acute and often self-limiting course, it is likely that components of the innate immune system play a central part in controlling virus replication and determining clinical outcome. Natural killer (NK) cells are innate lymphocytes with notable activity against a broad range of viruses, including RNA viruses ${ }^{1,2}$. NK cell function may be altered during COVID-19 despite increased representation of NK cells with an activated and adaptive phenotype ${ }^{3,4}$. Here we show that a decline in viral load in COVID-19 correlates with NK cell status and that NK cells can control SARS-CoV-2 replication by recognizing infected target cells. In severe COVID-19, NK cells show defects in virus control, cytokine production and cell-mediated cytotoxicity despite high expression of cytotoxic effector molecules. Single-cell RNA sequencing of NK cells over the time course of the COVID-19 disease spectrum reveals a distinct gene expression signature. Transcriptional networks of interferon-driven NK cell activation are superimposed by a dominant transforming growth factor- $\beta$ (TGF $\beta$ ) response signature, with reduced expression of genes related to cell-cell adhesion, granule exocytosis and cell-mediated cytotoxicity. In severe COVID-19, serum levels of TGF $\beta$ peak during the first two weeks of infection, and serum obtained from these patients severely inhibits NK cell function in a TGF $\beta$-dependent manner. Our data reveal that an untimely production of TGF $\beta$ is a hallmark of severe COVID-19 and may inhibit NK cell function and early control of the virus.
The role of NK cells during SARS-CoV-2 infection remains unknown. We wondered whether we could find differences in the decline in SARS-CoV-2 load between patients admitted to hospital with normal $(>40$ NK cells per $\mu \mathrm{l})$ or low $(\leq 40$ NK cells per $\mu \mathrm{l})$ NK cell counts. There was an overall pattern of a faster decline in viral load in patients with normal NK cell counts (Fig. 1a, b) across patient groups with different overall clinical statuses (Extended Data Fig. 1a, Supplementary Table 3). Of note, such a negative correlation between NK cell counts (early during the infection) and viral load was not found for T cells or B cells (Extended Data Fig. 1a). Similarly, a more rapid decline in viral load over time was associated with a more rapid increase in NK cells and vice versa (Extended Data Fig. 1b). Overall, the availability of NK cells early during the course of COVID-19 correlated with a lower abundance of SARS-CoV-2 viral RNA.
We investigated whether NK cells can directly control SARS-CoV-2 (B.1 lineage) replication in an infected human lung epithelial cell line (Calu-3) or in kidney epithelial cells (Vero E6). At the time of infection, highly purified NK cells from healthy donors were added to infected Calu-3 and Vero E6 cells and the intracellular viral load was measured. NK cells reduced viral replication in a dose-dependent manner (Fig.1c,d), a finding that was confirmed with a second virus variant (B.1.351 lineage; Extended Data Fig. 1c). NK cells are often activated during viral infections ${ }^{5}$, but NK cells isolated from patients admitted to hospital with COVID-19 were significantly less effective in reducing the viral load compared with NK cells from healthy donors (Fig. 1e,f).

NK cell recognition of virus-infected cells is determined by interactions between activating and inhibitory NK cell receptors and their ligands on target cells ${ }^{6}$. The large reduction in viral replication 


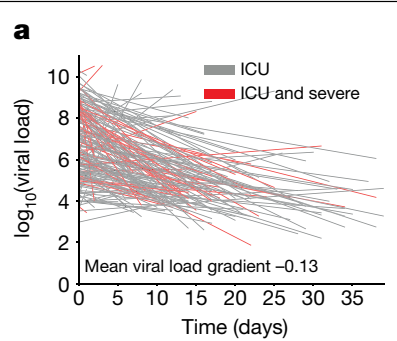

b
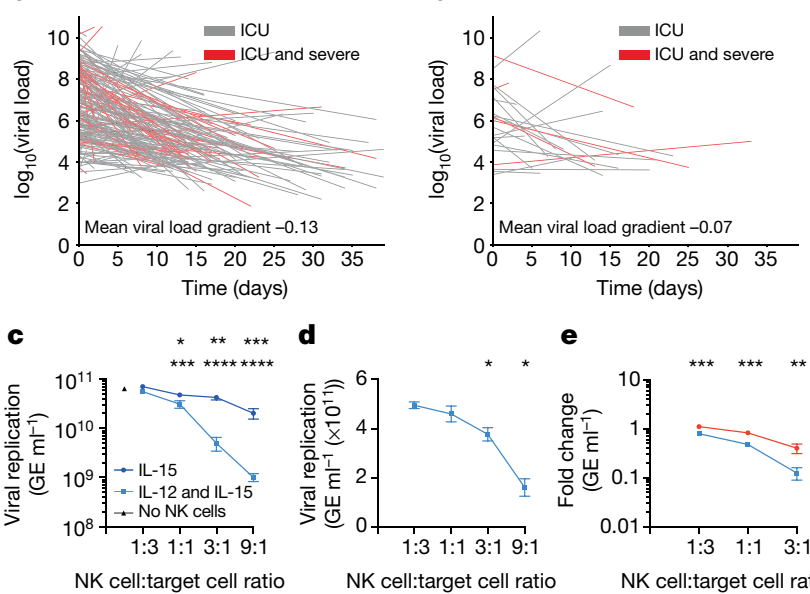

e
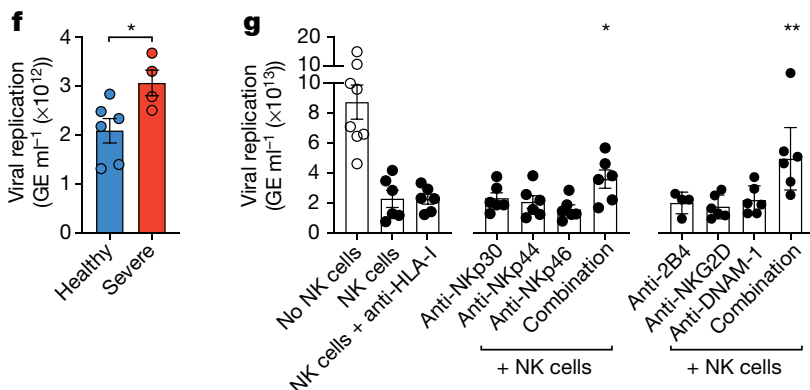

Fig. 1 | NK cells from patients with severe COVID-19 fail to control SARS-CoV-2 replication. a, b. Temporal viral load regression for patients with $>40$ NK cells per $\mu \mathrm{l}(\mathbf{a} ; n=183)$ or $\leq 40 \mathrm{NK}$ cells per $\mu \mathrm{l}(\mathbf{b} ; n=23)$ at first count (Methods). The $x$-axis indicates time since the first measurement. The analysis included 206 patients in the intensive care unit (ICU) (Extended Data Fig.1), of which 47 patients had severe COVID-19 (highlighted in red; Supplementary Table 3). c, d, Vero E6 cells (c) or Calu-3 cells (d) were infected with SARS-CoV-2 (B.1 lineage). At $1 \mathrm{~h}$ after infection, NK cells from healthy donors activated for $24 \mathrm{~h}$ in vitro as indicated with interleukins were added. Viral replication was measured $12 \mathrm{~h}$ later as genome equivalents (GE) per ml (GE ml ${ }^{-1}$; target cells co-cultured with NK cells versus cultured alone (c) or versus NK cell:target cell ratio 1:3 (d), $n=4$ donors, no NK cells $n=5$ samples). e, f, Vero E6 cells (e) or Calu- 3 cells (f) were infected with SARS-CoV- 2 and co-cultured as described above with NK cells from either healthy donors $(n=8(\mathbf{e})$ or $6(\mathbf{f}))$ or patients with severe COVID-19 $(n=6$ (e) or $4(\mathbf{f}))$. Viral load as the fold-change of Vero E6 cells cultured alone versus co-cultured with NK cells was determined (e; pooled data from two independent experiments). For f, a 1:1 NK cell:target cell ratio was used. g, Calu-3 cells were infected and co-cultured as above with IL-12- and IL-15-activated NK cells in a 3:1 NK cell:target cell ratio. Before co-culture, NK cells were incubated with the indicated neutralizing receptor antibodies. Each filled dot represents viral replication of target cells co-cultured with NK cells from an individual donor (2B $4 n=4$, all others $n=6$, no NK cells $n=8$ samples, indicated group versus NK cells only). Graphs display mean \pm s.e.m. Two-sided Mann-Whitney $U$-test $(\mathbf{f}, P=0.038 ; \mathbf{g}, P=0.041$ and $P=0.0043)$. ${ }^{*} P \leq 0.05$, ${ }^{* *} P \leq 0.01,{ }^{* * *} P \leq 0.001,{ }^{* * * *} P \leq 0.0001$.

induced by NK cells could not be further enhanced by HLA blockade, which suggests that infected Calu-3 cells do not appreciably inhibit NK cells through HLA-I-specific inhibitory receptors (Fig. 1g). Uninfected Calu-3 cells were poor targets of NK cells (Extended Data Fig.1d). Blockade of single, activating NK cell receptors did not impair virus control, whereas simultaneous blockade of all three natural cytotoxicity receptors (NKp30, NKp44 and NKp46) or of 2B4, NKG2D and DNAM-1 led to a significant increase in virus replication (Fig. 1g). Collectively, our data demonstrate that NK-cell-mediated control of SARS-CoV-2 replication in infected target cells requires redundant recognition by activating NK cell receptors. This process is impaired in infected cells treated with NK cells isolated from patients admitted to hospital with COVID-19.

\section{Impaired NK cell function during COVID-19}

We set out to study NK cell effector functions in detail in patients with COVID-19 across the disease spectrum and time (Fig. 2a). Patients with non-COVID-19 flu-like illness (FLI), ambulant patients with COVID-19 and patients with moderate COVID-19 disease severity had normal frequencies of CD56 ${ }^{\mathrm{dim}}$ NK cells. By contrast, patients with a severe course of COVID-19 had reduced frequencies of both CD56 ${ }^{\text {dim }}$ and CD $56^{\text {bright }} \mathrm{NK}$ cells and of innate lymphoid cells during the first weeks after symptom onset (Extended Data Fig. 2).

Previous data regarding the expression profile of cytotoxic molecules in COVID-19 were inconclusive ${ }^{3,4,7,8}$. We found a significant and early upregulation of perforin and granzyme $B$ both in ambulant patients and in hospitalized patients with COVID-19 (Fig. 2b, c, Extended Data Fig. $3 a-c)$, which is an early sign of NK cell activation that is observed in the context of various other viral infections ${ }^{5,9-11}$. NK cells isolated during the first two weeks after symptom onset from hospitalized and, to a lesser extent, from ambulant patients with COVID-19 showed impairments in cell-mediated cytotoxicity despite the high levels of perforin and granzyme B expression (Fig. 2d). Such a reduced cytotoxic activity of NK cells was not found in patients with FLI. Given the apparent paradox of high levels of cytotoxic molecule expression and low cytotoxic function, we analysed the release of cytotoxic granules. NK cells from healthy donors, patients with FLI and ambulant patients with COVID-19 did not show differences in degranulation. By contrast, NK cells from hospitalized patients with COVID-19 showed impaired degranulation (Fig. 2e, Extended Data Fig. 3d). One of the first steps during interactions between cognate NK cells and target cells is the formation of cellular conjugates, and the establishment of such conjugates was reduced for NK cells from patients with severe COVID-19 (Fig. 2f, g). Of note, reduced cell-cell interactions and degranulation were not a consequence of a reduced expression of activating NK cell receptors in severe COVID-19 (Extended Data Fig. 3e, f).

NK cells from ambulant patients with COVID-19 showed an increased production of interferon- $\gamma$ (IFN $\gamma$ ), whereas NK cells from patients with severe COVID-19 produced only low levels of IFN $\gamma$ and tumour necrosis factor (TNF) ${ }^{12}$ (Extended Data Fig. 3g, h). The T-box transcription factor T-bet coordinates NK cell effector programmes, including the expression of granzyme B, IFN $\gamma$ and perforin ${ }^{13}$. T-bet was upregulated in NK cells from patients with FLI and its expression was maintained in ambulant patients with COVID-19. By contrast, T-bet was substantially suppressed at all time points in NK cells from hospitalized patients with COVID-19 (Extended Data Fig. 3a, i). These data cannot be easily explained by differences in age because we did not find strong negative correlations between age and any of the NK cell readouts (Extended Data Fig. 4). Reductions in NK-cell-mediated cytotoxicity and effector programmes were also not caused by dexamethasone treatment, as comparable data were obtained for samples taken during the first wave of COVID-19 (March to April 2020), a period when dexamethasone was not administered to patients (Fig. 2e).

\section{Single-cell RNA sequencing atlas of NK cells}

We used single-cell RNA sequencing (scRNA-seq) to generate a time-resolved, droplet-based single-cell transcriptomics atlas of peripheral blood NK cells from the following individuals: patients with severe COVID-19; outpatients with oligosymptomatic SARS-CoV-2 infection; and healthy donors (Fig. 3a). Using the gating strategy depicted in Extended Data Fig. 5a, 80,325 single NK cell transcriptomes were captured. Using uniform manifold approximation and projection for dimension reduction (UMAP), we identified seven transcriptionally 


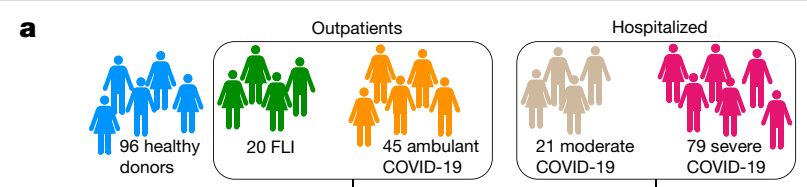

Longitudinal sampling of blood NK cells $(n=365$ COVID-19) NK cell function

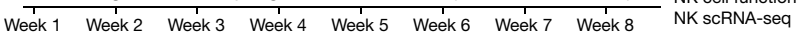
b
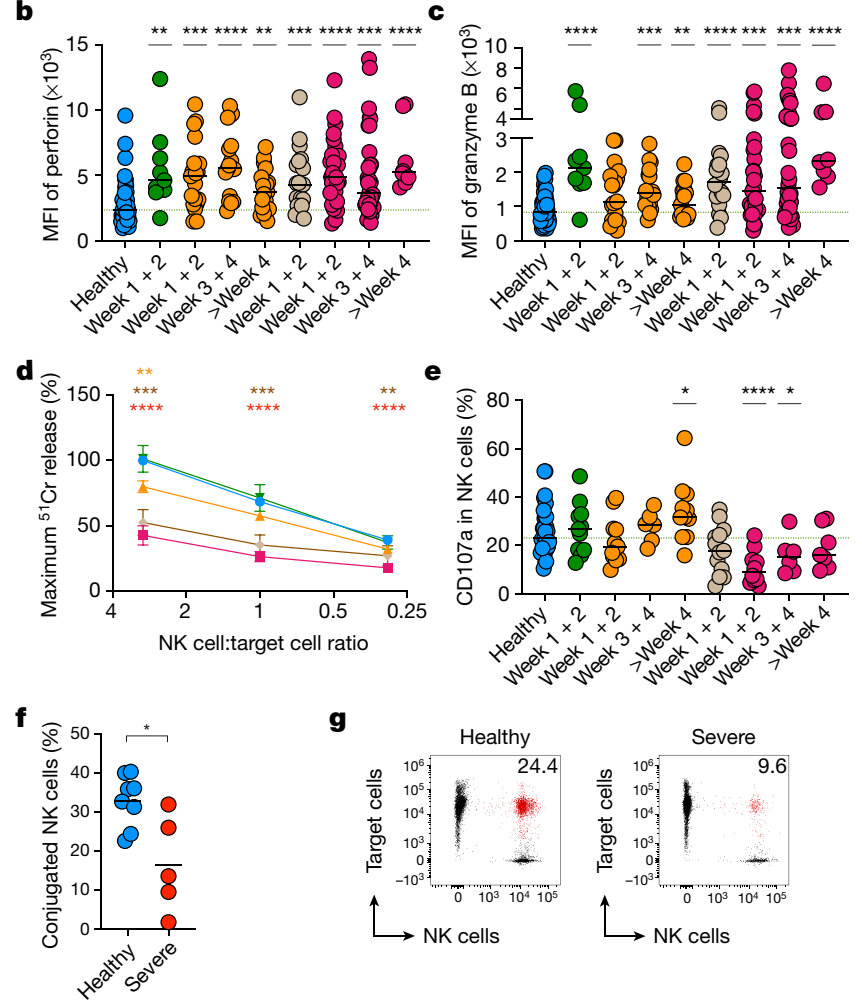

g
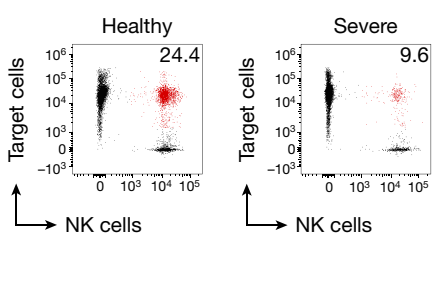

Fig. 2 | Impaired NK-cell-mediated cytotoxicity during severe COVID-19. a, Overview of the study design. b, c, Mean fluorescence intensity (MFI, median) of perforin (b) and granzyme B (c) in CD56 ${ }^{\mathrm{dim}} \mathrm{NK}$ cells. Independent measurements from 44 (b) or 50 (c) healthy donors, 9 patients with FLI, and patients with COVID-19 ( 24 ambulant, $n=56 ; 17$ moderate, $n=19 ; 30$ severe, $n=73$ ) obtained $1-8$ weeks after the onset of symptoms. d, Specific lysis (mean \pm s.e.m.) of K562 target cells by NK cells from the indicated donors obtained within the first 2 weeks after symptom onset was determined in a ${ }^{51} \mathrm{Chromium}\left({ }^{51} \mathrm{Cr}\right)$ release assay. Data represent pooled data of $12 \mathrm{independent}$ experiments using 18 healthy donors ( $n=38$ independent measurements), 8 patients with FLI $(n=8)$ and 28 patients with COVID-19 ( 7 ambulant, $n=7 ; 14$ moderate, $n=15 ; 10$ severe, $n=10$ ). e, PBMCs from the indicated donor groups were co-cultured for $4 \mathrm{~h}$ with $\mathrm{K} 562$ cells and the percentage (median) of $\mathrm{CD}_{107 \mathrm{a}^{+}} \mathrm{NK}$ cells was measured. Patients receiving corticosteroid treatment were excluded from the analysis. Data depict independent measurements across the disease course ( 29 healthy, $n=29 ; 11 \mathrm{FLI}, n=11 ; 21$ ambulant COVID-19, $n=28 ; 13$ moderate COVID-19, $n=13 ; 16$ severe COVID-19, $n=22$ ). $\mathbf{f}, \mathbf{g}$, Quantification (f, mean) and representative flow cytometry plots ( $g$ ) of conjugation of NK cells from healthy donors $(n=8)$ and from patients with severe COVID-19 $(n=5)$ with target cells. Numbers indicate the frequency in the percentage of NK cells conjugated to target cells out of all NK cells (pooled data from two independent experiments). For b, c and e, statistical analysis (indicated group versus healthy) was performed using one-way analysis of variance (ANOVA) followed by a two-sided Mann-Whitney $U$-test. The dashed line indicates median frequency or MFI of NK cells from healthy donors. For $\mathbf{d}$ and $\mathbf{f}$, two-sided Mann-Whitney $U$-test was used $(\mathbf{f}, P=0.03)$.

distinct clusters of cells ${ }^{14}$ (Fig. 3a). A small number (1,375 cells) of contaminating non-NK cells was found in cluster 6; therefore this cluster was excluded from further analysis. Cells in the remaining clusters ( $0-5)$ expressed genes that define conventional NK cells, including surface markers (Extended Data Fig. 5b), effector molecules, such as PRF1, GNLY, $G Z M B, G Z M H$ and $G Z M M$, and $T B X 21$ (which encodes T-bet) ${ }^{15,16}$ (Extended Data Fig. 5b-f). Cluster 2 represented CD56 ${ }^{\text {bright }} N K$ cells characterized by high expression of IL7R, SELL, XCL1, LTB and GZMK and low expression of $C D 16 O$ (refs. ${ }^{16,17}$ ) (Extended Data Fig. 5b,c). Cluster 0 contained cells with high expression of NK cell effector molecules and of TBX21, but with low expression of NCAM1, and we therefore identified these as a subset of CD56 ${ }^{\mathrm{dim}} \mathrm{NK}$ cells. Cluster 1 was closely related to cluster 0. Among the few differentially expressed genes were CD96 and KLRG1, both of which have been linked to NK cell maturation and functional exhaustion $^{18,19}$. In addition, they showed reduced expression of most NK effector genes, thus they demarcate late effector NK cells that may be reduced in function (Extended Data Fig. 5d). Within CD56 ${ }^{\mathrm{dim}} \mathrm{NK}$ cells, a third cluster (cluster 3 ) was discriminated with low expression of effector molecules (GZMB, GZMH and GZMM; Extended Data Fig. 5d). This NK cell subset may correspond to the previously described 'terminally differentiated' $\mathrm{CD} 56^{\mathrm{dim}} \mathrm{NK}$ cells that are in a post-activation state ${ }^{16,20}$.

Similar to CD56 ${ }^{\text {bright }} \mathrm{NK}$ cells, cells in cluster 4 were characterized by high expression of $I L 7 R$ and $G Z M K$, but expressed low levels of XCL1 and SELL (Extended Data Fig. 5c) and corresponded to "transitional NK cells"16. Cluster 4 also contained cells that express the activating NK cell receptor KLRC2 (which encodes NKG2C; Extended Data Fig. 5e). Low expression levels of NKG2A (encoded by $K L R C 1$ ) and of the signalling adaptor molecule FceR $\gamma$ (encoded by FCER1G) corroborated the enrichment of adaptive NK cells in cluster 4 (ref. ${ }^{21}$ ). Thus, cluster 4 is heterogenous and represents transitional and adaptive NK cells. Cluster 5 represented proliferating NK cells identified by the high expression of MKI67 and of genes controlling the cell cycle (Extended Data Fig. 5e, g).

An analysis of the representation of each NK cell cluster across COVID-19 disease states revealed dynamics in NK cell differentiation. In severe COVID-19, we observed a significant increase in proliferating (cluster 5) NK cells (Fig. 3b), and this result was in line with flow cytometry data (Extended Data Fig. 5h). Although the frequency of cells belonging to cluster 1 (late effector) NK cells was reduced in patients with severe COVID-19, these patients had increased levels of terminally differentiated cluster 3 NK cells (Fig. 3b). Although not statistically significant, adaptive NK cells (cluster 4 ) were reproducibly increased in severe COVID-19, results that are in line with previous data ${ }^{3}$.

To investigate whether COVID-19 introduces changes in the dynamics of NK cell differentiation, we used monocle pseudotime trajectory analysis ${ }^{22}$, which connects related clusters to construct differentiation trajectories (Fig. 3c, d, Extended Data Fig. 6a-d). We defined cluster 2 CD56 ${ }^{\text {bright }}$ NK cells as the root of the progression trajectory. The pseudotime model predicted that CD56 $6^{\text {bright }}$ NK cells (cluster 2)-through a proliferative state in the left leaf (cluster 5)-differentiate into effector NK cells (cluster 0: CD56 ${ }^{\mathrm{dim}} \mathrm{NK}$ effector I cells). Next, by gradually decreasing effector molecule expression, the effector NK cells differentiate into cluster 1 CD56 ${ }^{\text {dim }}$ effector II cells until reaching the terminally differentiated state (cluster 3 ), which dominates the distal right leaf of the differentiation trajectory (Fig. 3c, d, Extended Data Fig. 6a, b). Along the pseudotime trajectory, major differentiation states were CD56 $6^{\text {bright }}$ NK cells (state 12: high expression of IL7RA and GZMK), proliferating NK cells (state 1: MKI67 high), effector NK cells (states 2-7; with graded levels of effector genes such as PRF1) and finally terminally differentiated NK cells (states 8 and 9) (Extended Data Fig. 6c, d). This differentiation trajectory was generally conserved in patients with COVID-19. However, patients with particularly severe COVID-19 had increased levels of proliferating NK cells that fed into one root of the differentiation trajectory and had an accumulation of terminally differentiated NK cells at the distal end of the trajectory (Extended Data Fig. 6a-d).

\section{A TGF $\beta$ signature is a hallmark of severe COVID-19}

We interrogated our dataset for enrichment of genes that may affect NK cell effector functions and applied gene set enrichment analysis (GSEA) 

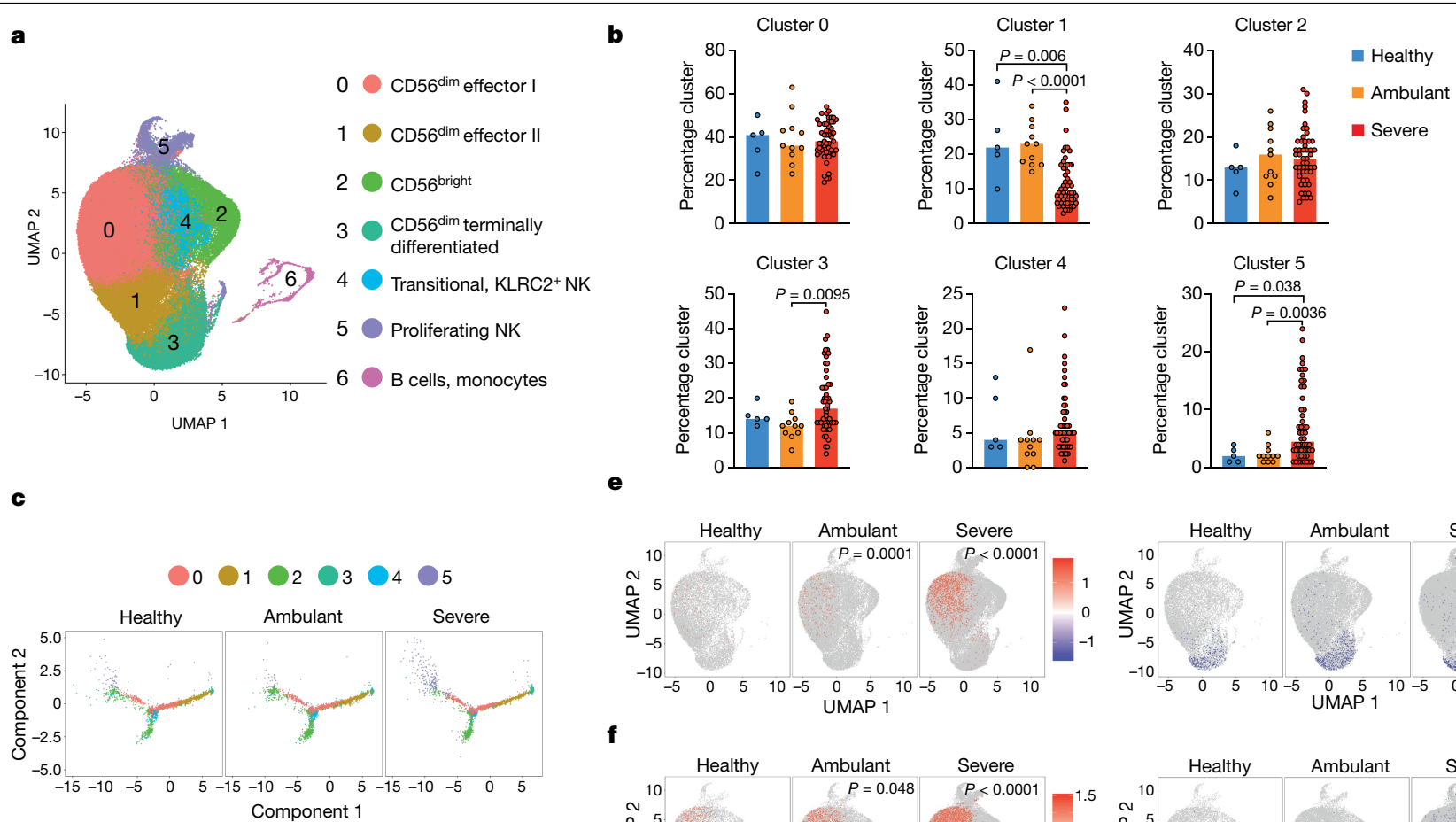

e
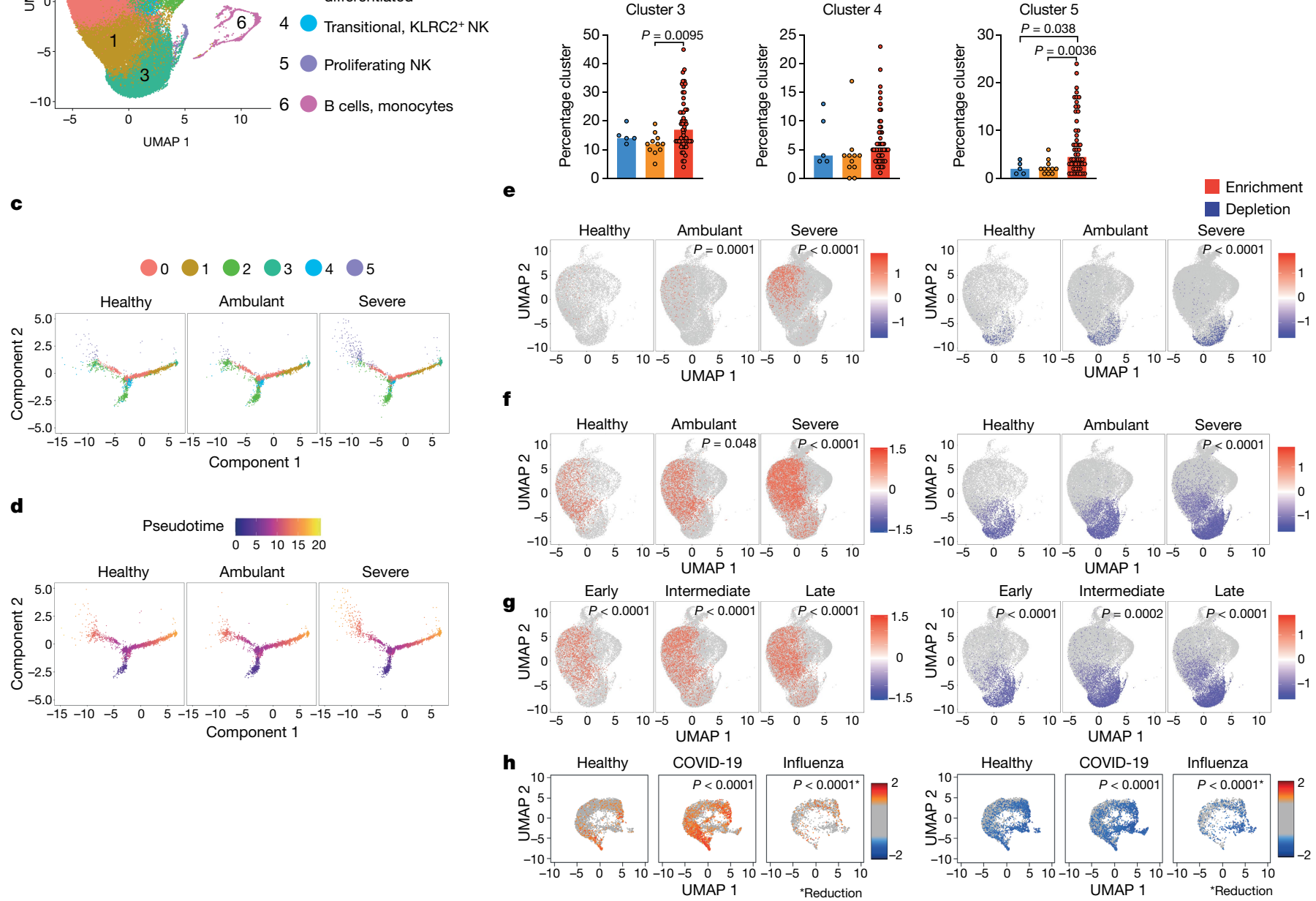

Fig. 3 | A TGF $\beta$ response signature is a hallmark of NK cells in severe COVID-19 but not in severe influenza. $\mathbf{a}-\mathbf{g}$, scRNA-seq of highly purified peripheral blood NK cells of 5 healthy donors $(n=5)$ and patients with COVID-19 from day 2 to day 68 after symptom onset (11 ambulant patients $(n=11)$ and 13 patients with severe COVID-19 $(n=52)$ (Supplementary Tables $1-3)$. The gating strategy is depicted in Extended Data Fig. 5a.a, UMAP representation of 80,325 sorted NK cells from all samples $(n=68)$. Colours indicate unsupervised clustering. b, Percentage (median) of cells allocated to each cluster in the indicated groups. Each dot represents one sample subjected to scRNA-seq. $P$ values determined by one-way ANOVA followed by two-sided Mann-Whitney $U$-test.c, d, Pseudotime trajectories (c) and representation of the individual NK cell clusters in trajectories (d) of 11,613 randomly selected NK cell transcriptomes from all groups. $\mathbf{e}-\mathbf{g}$, Single-cell GSEA of indicated gene sets was projected on the UMAP analysis (clusters 0,1 and 3 only). Single cells with enriched gene expression are displayed as red dots (left), cells with depletion of the genes are displayed as blue dots (right). All sequenced NK cells per group are displayed as grey dots in the background. e, Hallmark IFN $\alpha$ response GSEA. $\mathbf{f}, \mathbf{g}$, Enrichment of TGF $\beta$-induced genes (f, left) and depletion of TGF $\beta$-suppressed genes (f, right) in the indicated groups and across the course of severe COVID-19 (g; early: <day 14 after symptom onset; intermediate: days 15-28, late:>day 28).h, NK cells were extracted from a publicly available single-cell dataset of PBMCs from healthy donors and from patients with COVID-19 or with severe influenza ${ }^{30}$ and a single-cell GSEA of the NK-cell-specific TGF $\beta$ response gene set was performed as described in e. GSEA $P$ values were calculated using two-sided Fisher's exact test comparing the indicated groups with the left-sided (in e, early was compared to healthy). ${ }^{*} P$ value describes a reduction in enrichment or depletion. to the effector NK cell clusters 0,1 and 3 . In patients with COVID-19, there was a significant enrichment of 'Hallmark IFN $\alpha$ response genes' (Fig. 3e) and 'Hallmark IFN $\gamma$ response genes' (Extended Data Fig. 6e) predominantly in the NK effector cluster 0 . This result was in line with increased virus-induced type I and type II interferon levels observed in the serum of patients with COVID-19 (Extended Data Fig. 6f). We also confirmed ${ }^{3}$ an increased expression of markers linked to NK cell activation and differentiation (for example, CD69 and CD57) in hospitalized patients with COVID-19 (Extended Data Fig. 6g-i). Among the enriched functional networks in NK cells isolated from patients with COVID-19 were several gene sets related to cellular metabolism and translation. Together, these results demonstrate that there are substantial changes in cellular activation in the context of SARS-CoV-2 infections (Extended Data Fig. 7a).

We next performed a Kyoto Encyclopedia of Genes and Genomes (KEGG) pathway analysis. We noted that among the transcriptional networks significantly enriched in effector NK cells from ambulant patients with COVID-19 and in particular from patients with severe COVID-19, was the pathway 'intestinal immune network for IgA production' (Extended 


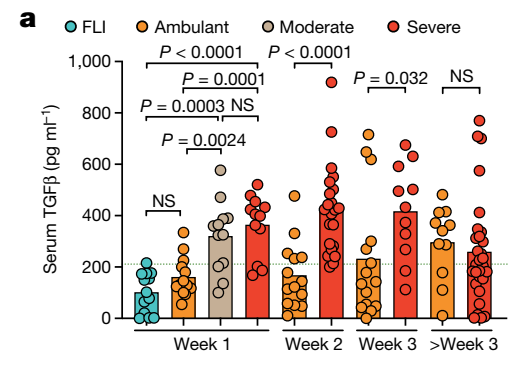

b

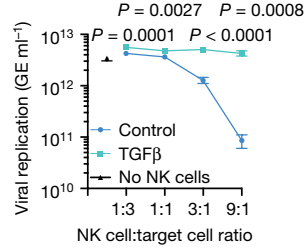

c

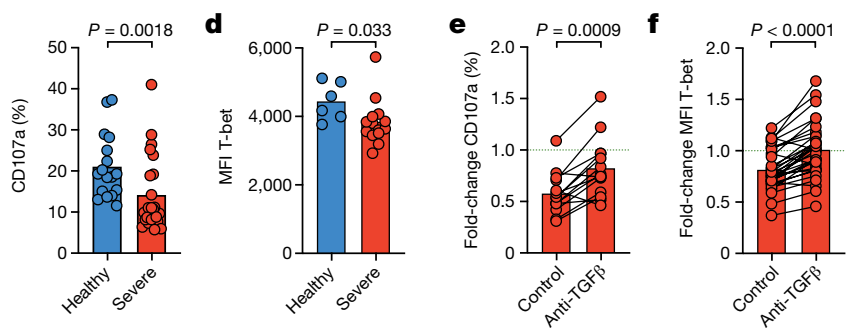

Fig. 4 | Serum of patients with severe COVID-19 inhibits NK cell function in a TGF $\beta$-dependent manner. a, Serum levels of active TGF $\beta$. Independent measurements (mean) from 13 patients with $\mathrm{FLI}(n=13)$ and 66 patients with COVID-19 (30 severe, $n=74 ; 7$ moderate, $n=12 ; 39$ ambulant, $n=53$ ) at indicated time points after symptom onset (group $>3$ weeks contains samples from weeks 4 and 5). The dashed line indicates the median TGF $\beta$ serum level of 34 healthy donors. Patients receiving corticosteroids were excluded. b, NK cells from healthy donors were cultured in medium containing IL-12 and IL-15 with (green) or without TGF $\beta$ (blue) and co-cultured with SARS-CoV-2-infected Vero E6 cells. Viral load (mean \pm s.e.m.) was measured $12 \mathrm{~h}$ later. Each data point represents NK cells from one individual donor (E:T 9:1 $n=3$, all others $n=4$, no NK cells $n=11$ samples). c, d, Sorted NK cells from 3-9 healthy donors were cultured in medium containing either serum from a healthy donor or serum from a patient with severe COVID-19 $(n=9$ (c) or 7 (d)) and the frequency of $\mathrm{CD} 107 \mathrm{a}^{+} \mathrm{NK}$ cells (mean) after co-culture with $\mathrm{K} 562$ (c) or the MFI of T-bet (d, mean) was determined. Each dot represents NK cells of one healthy donor cultured with serum of another healthy donor (blue; $n=17$ (c) or $6(d)$ ) or of one patient with severe COVID-19 (red; $n=24$ (c) or 14 (d)).e,f, Sorted NK cells from 3-8 healthy donors were cultured in medium alone or with serum from patients with severe COVID-19. In a second condition, patient sera were pre-incubated with anti-TGF $\beta$ and then added as described above. The frequency of CD107a ${ }^{+}$ NK cells (e, mean, $n=14)$ and the MFI of T-bet $(\mathbf{f}$, mean, $n=32)$ were determined. Shown is the fold-change frequency or MFI between NK cells cultured in sera from patients (with or without prior anti-TGF $\beta$ treatment) and NK cells cultured in medium alone. For e, $\mathbf{f}$, two-sided Wilcoxon matched-pairs rank test was used. For a-d, two-sided Mann-Whitney $U$-test was used.

Data Fig. 7b). IgA class switching is strictly controlled by TGF $\beta^{23,24}$, and many genes in this KEGG pathway are direct targets of TGF $\beta$ signalling, a cytokine that suppresses NK cell function ${ }^{25,26}$. Even terminally differentiated (cluster 3 ) NK cells, which were substantially depleted in gene sets regulated by IFN $\alpha$ or cellular metabolism (Extended Data Fig. 7a), were enriched for genes of this KEGG pathway (Extended Data Fig. 7b). Because the transcriptional changes instructed by TGF $\beta$ in NK cells are not known at single-cell resolution, we generated a scRNA-seq dataset of highly purified peripheral blood NK cells from healthy donors cultured in the presence or absence of TGF $\beta$. UMAP analysis of the two treatment groups showed a large effect of TGF $\beta$ on the transcriptional state of NK cells that was highly reproducible across individual donors (Extended Data Fig. 8a). An analysis of differentially expressed genes revealed that the majority of genes were negatively regulated by TGF $\beta$ in their expression, including TBX21 and STAT1 and several effector genes such as PRF1 and granzyme family members (Extended Data Fig. $8 \mathrm{~b}$ ). The expression of a small group of genes was induced by exposure to TGF $\beta$, including genes that encode the chemokine receptor CXCR4 or the transcription factor EOMES, which are both known TGF $\beta$ target genes (Extended Data Fig. 8b). Within the cells that were exposed to
TGF $\beta$ (Extended Data Fig. 8c, red), we found that various pathways that are central to granule exocytosis ${ }^{27}$ were negatively regulated by TGF $\beta$ (Extended Data Fig. 8d).

Using this established TGF $\beta$ signature, we interrogated the scRNA-seq dataset from patients with COVID-19 for changes in the expression of TGF $\beta$-controlled genes. TGF $\beta$-controlled genes were expressed at a low level in NK cells from healthy donors, but a substantial enrichment in TGF $\beta$ response genes was noted in effector NK cell clusters from patients with severe COVID-19 (Fig.3f). Of note, changes in the expression of TGF $\beta$-controlled genes were already detectable during the very early stages of COVID-19 and increased during the course of infection (Fig. 3g). Indeed, the expression of canonical TGF $\beta$ target genes such as EOMES and ITGAE was upregulated in NK cells from patients with severe COVID-19 (Extended Data Fig. 8e,f). Our data demonstrate that there is a progressive and long-lasting reprogramming of NK cells by TGF $\beta$ during COVID-19 that starts within the first days after symptom onset and is commensurate with disease severity.

In ambulant patients with COVID-19, gene sets associated with cell adhesion and NK cell cytotoxicity were slightly upregulated in some NK cells. However, substantial downregulation was found in most effector NK cells in severe COVID-19 (Extended Data Fig. 8g). Of note, among the genes substantially downregulated in patients with severe COVID-19 was ITGB2, which encodes the $\beta_{2}$-integrin (also known as CD18). This integrin associates with the $\alpha$-integrins CD11a to CD11d to generate functional integrin receptors (Extended Data Fig. 8h) and is involved in NK-cell-mediated cytotoxicity ${ }^{28,29}$. This may provide a molecular explanation for the substantial failure of NK cells from patients with severe COVID-19 to form conjugates with target cells. Collectively, NK cells from patients with severe COVID-19 show a gene signature characterized by IFN-controlled cell activation programmes (for example, upregulation of perforin, granzyme B and CD69) superimposed by strong and long-lasting TGF $\beta$-controlled transcriptional reprogramming, in particular the downregulation of genes linked to NK-cell-mediated cytotoxicity.

We wondered whether reprogramming of NK cells by TGF $\beta$ is a common event in pneumonia. We extracted NK cell data from a previously published scRNA-seq dataset of peripheral blood mononuclear cells (PBMCs) from patients with COVID-19 and from patients with influenza pneumonia ${ }^{30}$. This analysis revealed a strong enrichment of TGF $\beta$-controlled genes in NK cells from patients with COVID-19 but not from patients with influenza pneumonia (Fig. 3h). To explore whether TGF $\beta$ signatures can also be found in lung NK cells, we analysed a single-nucleus RNA-seq dataset of post-mortem lung tissue samples from patients with severe COVID-19 or with SARS-CoV-2-negative pneumonia $^{31}$. The dataset enabled the analysis of single-cell transcriptomes of lung NK cells (Extended Data Fig. 9a-c). GSEA revealed that lung NK cells from patients with severe COVID-19 pneumonia but not those from patients with SARS-CoV-2-negative pneumonia were significantly enriched in TGF $\beta$-controlled genes (Extended Data Fig. 9d).

\section{Untimely peak of serum TGF $\beta$ in severe COVID-19}

Our transcriptional and functional data suggested that TGF $\beta$ is produced early in the phase of SARS-CoV-2 infection, particularly in the context of severe COVID-19. Patients with FLI and ambulant patients with COVID-19 did not show increased serum TGF $\beta$ within the first week after symptom onset, whereas hospitalized patients with COVID-19 had significantly increased TGF $\beta$ serum levels at week 1 that peaked at week 2 (Fig. 4a). Ambulant patients with COVID-19 showed only a small increment in serum TGF $\beta$ at later time points of the infection ( $>3$ weeks after symptom onset). To obtain insights into the cellular sources of TGF $\beta$, we analysed the lung single-nucleus RNA-seq dataset for TGFB transcripts and for transcripts associated with TGF $\beta$ bioactivity. In comparison to non-COVID-19 pneumonia illnesses, we observed a large increase in $T G F B 1$ and $T G F B 2$ expression in type 1 alveolar epithelial 
cells, fibroblasts, myofibroblasts, endothelial cells and myeloid cells (Extended Data Fig. 9e,g). Expression levels of various genes required for the bioactivity of TGF $\beta$ (such as $L T B P 3, L A P 3$ and MMP2) were also increased in patients with COVID-19 (Extended Data Fig. 9f). Of note, some of the clusters with the highest expression of TGFB1, LAP3 and $M M P 28$ contained SARS-CoV-2 transcripts (that is, were infected) (Extended Data Fig. 9h). Thus, the early TGF $\beta$ peak in hospitalized patients with COVID-19 is closely correlated to the early impairment of NK cell effector programmes.

We wondered whether exposure of NK cells to TGF $\beta$ has any effect on their capacity to control viral replication. TGF $\beta$ completely abrogated the NK cell effector programme, that is, NK-cell-mediated control of SARS-CoV-2 replication in vitro (Fig. 4b), cell-mediated cytotoxicity, degranulation in response to target cells and cytokine production (Extended Data Fig. 10a-d). TGF $\beta$ treatment of stimulated NK cells also led to the downregulation of T-bet, whereas the canonical TGF $\beta$ target EOMES was upregulated (Extended Data Fig. 10e, f).

NK cells from healthy donors that were exposed to serum from patients with severe COVID-19, obtained during the first weeks after symptom onset, significantly inhibited NK cell degranulation and T-bet expression (Fig. 4c, d). This inhibitory effect was lost when sera was pretreated with a TGF $\beta$-blocking antibody with activity against TGF $\beta 1$, TGF $\beta 2$ and TGF $\beta 3$ (Fig. 4e, f, Extended Data Fig. 10g-i). By contrast, neutralization of interleukin-6 (IL-6), IL-10 or IL-15 did not restore NK cell degranulation or T-bet expression (Extended Data Fig. 10j-p), even though these cytokines have been previously linked to severe COVID-19 (refs. ${ }^{4,32}$ ). Thus, the untimely expression of TGF $\beta$ leads to suppressed NK cell function, which may reduce virus control and be detrimental in severe COVID-19.

Although TGF $\beta$ is thought to curtail excessive immune responsiveness and to restore or maintain immune homeostasis ${ }^{33}$, we now demonstrate that an untimely early production of TGF $\beta$ and associated NK cell dysfunction is a hallmark of severe COVID-19. Multilevel proteomics data support a specific dysregulation of TGF $\beta$ signalling by the SARS-CoV-2 ORF8 protein ${ }^{34}$ and we have previously shown that TGF $\beta$ impairs B-cell responses in the context of COVID-19 (ref. ${ }^{35}$ ). Two matrix metalloproteinase inhibitors (prinomastat and marimastat) that diminish TGF $\beta$ bioactivity strongly inhibited the replication of SARS-CoV- 2 but not of SARS-CoV ${ }^{34}$. TGF $\beta$-mediated impairment of NK cell function may also have an impact on other aspects of COVID-19. A dysregulated myeloid response is another hallmark of severe COVID-19 in which there is an inadequate production of inflammatory cytokines ${ }^{36,37}$. Lessons learnt from genetic syndromes of NK cell cytotoxic defects have implicated NK cells in the quality control of innate immune responses by curtailing excessive myeloid responses, thereby preventing immunopathology ${ }^{27,38}$. Another intriguing link is the role of NK cells in the control of fibrotic reprogramming and the elimination of pre-fibrotic cells that undergo a senescence programme $^{39}$. Thus, the inhibition of untimely TGF $\beta$ production and the promotion of NK cell function may positively affect SARS-CoV-2 control on multiple levels ${ }^{40}$.

\section{Online content}

Any methods, additional references, Nature Research reporting summaries, source data, extended data, supplementary information, acknowledgements, peer review information; details of author contributions and competing interests; and statements of data and code availability are available at https://doi.org/10.1038/s41586-021-04142-6.

1. Diaz-Salazar, C. \& Sun, J. C. Natural killer cell responses to emerging viruses of zoonotic origin. Curr. Opin. Virol. 44, 97-111 (2020).

2. Vivier, E., Tomasello, E., Baratin, M., Walzer, T. \& Ugolini, S. Functions of natural killer cells. Nat. Immunol. 9, 503-510 (2008).

3. Maucourant, C. et al. Natural killer cell immunotypes related to COVID-19 disease severity. Sci. Immunol. 5, eabd6832 (2020).
4. Mazzoni, A. et al. Impaired immune cell cytotoxicity in severe COVID-19 is IL-6 dependent. J. Clin. Invest. 130, 4694-4703 (2020).

5. Lucas, M., Schachterle, W., Oberle, K., Aichele, P. \& Diefenbach, A. Dendritic cells prime natural killer cells by trans-presenting interleukin 15. Immunity 26, 503-517 (2007).

6. Diefenbach, A. \& Raulet, D. H. Innate immune recognition by stimulatory immunoreceptors. Curr. Opin. Immunol. 15, 37-44 (2003).

7. Zenarruzabeitia, O. et al. T cell activation, highly armed cytotoxic cells and a shift in monocytes $\mathrm{CD} 300$ receptors expression is characteristic of patients with severe COVID-19. Front. Immunol 12, 655934 (2021).

8. Zheng, M. et al. Functional exhaustion of antiviral lymphocytes in COVID-19 patients. Cell. Mol. Immunol. 17, 533-535 (2020).

9. Shellam, G., Allan, J., Papadimitriou, J. \& Bancroft, G. Increased susceptibility to cytomegalovirus infection in beige mutant mice. Proc. Natl Acad. Sci. USA 78, 5104-5108 (1981).

10. Trinchieri, G. Biology of natural killer cells. Adv. Immunol. 47, 187-376 (1989).

11. Fehniger, T. A. et al. Acquisition of murine NK cell cytotoxicity requires the translation of a pre-existing pool of granzyme B and perforin mRNAs. Immunity 26, 798-811 (2007).

12. Varchetta, S. et al. Unique immunological profile in patients with COVID-19. Cell. Mol. Immunol. 18, 604-612 (2021).

13. Townsend, M. J. et al. T-bet regulates the terminal maturation and homeostasis of NK and Va14i NKT cells. Immunity 20, 477-494 (2004).

14. Becht, E. et al. Dimensionality reduction for visualizing single-cell data using UMAP. Nat. Biotechnol. 37, 38-44 (2019).

15. Freud, A. G. et al. NKp80 defines a critical step during human natural killer cell development. Cell Rep. 16, 379-391 (2016).

16. Yang, C. et al. Heterogeneity of human bone marrow and blood natural killer cells defined by single-cell transcriptome. Nat. Commun. 10, 3931 (2019).

17. Luetke-Eversloh, M., Killig, M. \& Romagnani, C. Signatures of human NK cell development and terminal differentiation. Front. Immunol. 4, 499 (2013).

18. Huntington, N. D. et al. NK cell maturation and peripheral homeostasis is associated with KLRG1 up-regulation. J. immunol. 178, 4764-4770 (2007).

19. Sun, H. et al. Human CD96 correlates to natural killer cell exhaustion and predicts the prognosis of human hepatocellular carcinoma. Hepatology 70, 168-183 (2019).

20. Crinier, A. et al. High-dimensional single-cell analysis identifies organ-specific signatures and conserved NK cell cubsets in humans and mice. Immunity 49, 971-986.e5 (2018).

21. Hammer, Q. et al. Peptide-specific recognition of human cytomegalovirus strains controls adaptive natural killer cells. Nat. Immunol. 19, 453-463 (2018).

22. Qiu, X. et al. Reversed graph embedding resolves complex single-cell trajectories. Nat. Methods 14, 979-982 (2017).

23. Coffman, R. L., Lebman, D. A. \& Shrader, B. Transforming growth factor beta specifically enhances $\operatorname{Ig} A$ production by lipopolysaccharide-stimulated murine B lymphocytes. J. Exp. Med. 170, 1039-1044 (1989).

24. Sonoda, E. et al. Transforming growth factor beta induces IgA production and acts additively with interleukin 5 for IgA production. J. Exp. Med. 170, 1415-1420 (1989).

25. Viel, S. et al. TGF- $\beta$ inhibits the activation and functions of NK cells by repressing the mTOR pathway. Sci Signal. 9, ra19 (2016).

26. Rook, A. H. et al. Effects of transforming growth factor beta on the functions of natural killer cells: depressed cytolytic activity and blunting of interferon responsiveness. J. Immunol. 136, 3916-3920 (1986).

27. Pachlopnik Schmid, J. et al. Inherited defects in lymphocyte cytotoxic activity. Immunol. Rev. 235, 10-23 (2010).

28. Kohl, S., Springer, T. A., Schmalstieg, F. C., Loo, L. S. \& Anderson, D. C. Defective natural killer cytotoxicity and polymorphonuclear leukocyte antibody-dependent cellular cytotoxicity in patients with LFA-1/OKM-1 deficiency. J. Immunol. 133, 2972-2978 (1984).

29. Riteau, B., Barber, D. F. \& Long, E. O. Vav1 phosphorylation is induced by $\beta 2$ integrin engagement on natural killer cells upstream of actin cytoskeleton and lipid raft reorganization. J. Exp. Med. 198, 469-474 (2003).

30. Lee, J. S. et al. Immunophenotyping of COVID-19 and influenza highlights the role of type I interferons in development of severe COVID-19. Sci. Immunol. 5, eabd1554 (2020).

31. Gassen, N. C. et al. SARS-CoV-2-mediated dysregulation of metabolism and autophagy uncovers host-targeting antivirals. Nat. Commun. 12, 3818 (2021).

32. Liu, C. et al. Time-resolved systems immunology reveals a late juncture linked to fatal COVID-19. Cell 184, 1836-1857.e22 (2021).

33. Travis, M. A. \& Sheppard, D. TGF- $\beta$ activation and function in immunity. Annu. Rev. Immunol. 32, 51-82 (2014).

34. Stukalov, A. et al. Multilevel proteomics reveals host perturbations by SARS-CoV-2 and SARS-CoV. Nature 594, 246-252 (2021).

35. Ferreira-Gomes, M. et al. SARS-CoV-2 in severe COVID-19 induces a TGF- $\beta$-dominated chronic immune response that does not target itself. Nat. Comm. 12, 1961 (2021).

36. Schulte-Schrepping, J. et al. Severe COVID-19 is marked by a dysregulated myeloid cell compartment. Cell 182, 1419-1440.e23 (2020).

37. Merad, M. \& Martin, J. C. Pathological inflammation in patients with COVID-19: a key role for monocytes and macrophages. Nat. Rev. Immunol. 20, 355-362 (2020).

38. van Dommelen, S. L. et al. Perforin and granzymes have distinct roles in defensive immunity and immunopathology. Immunity 25, 835-848 (2006).

39. Krizhanovsky, V. et al. Senescence of activated stellate cells limits liver fibrosis. Cell 134, 657-667 (2008)

40. Chen, W. A potential treatment of COVID-19 with TGF- $\beta$ blockade. Int. J. Biol. Sci. 16 1954-1955 (2020)

Publisher's note Springer Nature remains neutral with regard to jurisdictional claims in published maps and institutional affiliations.

(c) The Author(s), under exclusive licence to Springer Nature Limited 2021 
'Laboratory of Innate Immunity, Institute of Microbiology, Infectious Diseases and Immunology, Charité - Universitätsmedizin Berlin, Corporate Member of Freie Universität Berlin and Humboldt-Universität zu Berlin, Campus Benjamin Franklin, Berlin, Germany. ${ }^{2}$ Mucosal and Developmental Immunology, Deutsches Rheuma-Forschungszentrum (DRFZ), an Institute of the Leibniz Association, Berlin, Germany. ${ }^{3}$ Department of Microbiology and Hygiene, Labor Berlin, Charité-Vivantes, Berlin, Germany. ${ }^{4}$ Therapeutic Gene Regulation, Deutsches Rheuma-Forschungszentrum (DRFZ), an Institute of the Leibniz Association, Berlin, Germany. ${ }^{5}$ Institute of Virology, Charité - Universitätsmedizin Berlin, Corporate Member of Freie Universität Berlin and Humboldt-Universität zu Berlin, Campus Charité Mitte, Berlin, Germany. ${ }^{6}$ German Center for Infection Research (DZIF), Partner Site Berlin, Berlin, Germany. ${ }^{7}$ Centre for Pathogen Evolution, Department of Zoology, University of Cambridge, Cambridge, UK. ${ }^{8}$ Dermatological Allergology, Allergie-Centrum-Charité, Department of Dermatology and Allergy, Charité-Universitätsmedizin Berlin, Corporate Member of Freie Universität Berlin and Humboldt-Universität zu Berlin, Campus Charité Mitte, Berlin, Germany. ${ }^{9}$ Fraunhofer Institute for Translational Medicine and Pharmacology (ITMP) Allergology and Immunology, Berlin, Germany. ${ }^{10}$ Department of Anesthesiology and Intensive Care Medicine, Charité Universitätsmedizin Berlin, Corporate Member of Freie Universität Berlin and HumboldtUniversität zu Berlin, Campus Benjamin Franklin, Berlin, Germany. "Department of Pediatric Gastroenterology, Nephrology and Metabolic Diseases, Charité - Universitätsmedizin Berlin, Corporate Member of Freie Universität Berlin and Humboldt-Universität zu Berlin, Campus Virchow-Klinikum, Berlin, Germany. ${ }^{12}$ Institute of Physiology, Center for Space Medicine and Extreme Environments Berlin, Charité-Universitätsmedizin Berlin, Corporate Member of Freie Universität Berlin and Humboldt-Universität zu Berlin, Berlin, Germany. ${ }^{13}$ Department of Immunology, Labor Berlin, Charité-Vivantes, Berlin, Germany. ${ }^{14}$ Center for Digital Health, Berlin Institute of Health (BIH) and Charité-Universitätsmedizin Berlin, Corporate Member of Freie Universität Berlin and Humboldt-Universität zu Berlin, Berlin, Germany. ${ }^{15}$ Department of Neuropathology, Charité-Universitätsmedizin Berlin, Corporate Member of Freie Universität Berlin and Humboldt-Universität zu Berlin, Berlin, Germany. ${ }^{16}$ Institute of Medical Immunology, Charité - Universitätsmedizin Berlin, Corporate Member of Freie Universität
Berlin and Humboldt-Universität zu Berlin, Campus Virchow-Klinikum, Berlin, Germany. ${ }^{17}$ Department of Pediatric Respiratory Medicine, Immunology and Critical Care Medicine, Charité-Universitätsmedizin Berlin, Corporate Member of Freie Universität Berlin and Humboldt-Universität zu Berlin, Berlin, Germany. ${ }^{18}$ Department of Medicine (Gastroenterology, Infectious Diseases, Rheumatology), Charité - Universitätsmedizin Berlin, Corporate Member of Freie Universität Berlin, Humboldt-Universität zu Berlin and Berlin Institute of Health, Campus Benjamin FranklinBerlin, Germany. ${ }^{19}$ German Center for Lung Research (DZL), Associated Partner Berlin, Berlin, Germany. ${ }^{20}$ Laboratory of Mucosal Immunity, Institute of Microbiology, Infectious Diseases and Immunology, Charité Universitätsmedizin Berlin, Corporate Member of Freie Universität Berlin and HumboldtUniversität zu Berlin, Campus Benjamin Franklin, Berlin, Germany. ${ }^{21}$ Cancer Immunology Program, Peter MacCallum Cancer Centre, Melbourne, Victoria, Australia. ${ }^{22}$ Department of Experimental Medicine, University of Genoa, Genoa, Italy. ${ }^{23}$ Chronic inflammation in Childhood, Deutsches Rheuma-Forschungszentrum (DRFZ), an Institute of the Leibniz Association, Berlin, Germany. ${ }^{24}$ Department of Infectious Diseases and Respiratory Medicine, Charité - Universitätsmedizin Berlin, Corporate Member of Freie Universität Berlin and Humboldt-Universität zu Berlin, Berlin, Germany. ${ }^{25}$ Department of Tropical Medicine, Bernhard Nocht Institute for Tropical Medicine, and Department of Medicine I, University Medical Centre Hamburg-Eppendorf, Hamburg, Germany. ${ }^{26}$ Chronic Inflammation, Deutsches Rheuma-Forschungszentrum (DRFZ), an Institute of the Leibniz Association, Berlin, Germany. ${ }^{27}$ Belozersky Institute of Physico-Chemical Biology and Biological Faculty, M.V. Lomonosov Moscow State University, Moscow, Russia. ${ }^{28}$ Center for Precision Genome Editing and Genetic Technologies for Biomedicine, Engelhardt Institute of Molecular Biology, Russian Academy of Sciences, Moscow, Russia. ${ }^{29}$ Cell Biology, Deutsches Rheuma-Forschungszentrum (DRFZ), an Institute of the Leibniz Association, Berlin, Germany. ${ }^{30} \mathrm{BIH}$ Center for Regenerative Therapies (BCRT), Charité Universitätsmedizin Berlin, Berlin, Germany. ${ }^{31}$ These authors contributed equally: Mario Witkowski, Caroline Tizian, Marta Ferreira-Gomes. ${ }^{32}$ These authors jointly supervised this work: Mir-Farzin Mashreghi, Andreas Diefenbach. ${ }^{\bowtie}$ e-mail: mario.witkowski@ charite.de; andreas.diefenbach@charite.de 


\section{Article}

\section{Methods}

\section{Human participants}

The recruitment of study participants was approved by the Institutional Review Board of Charité (EA2/066/20, EA2/072/20, EAEA4/014/20 and EA2/092/20). Written informed consent was provided by all patients or legal representatives for participation in the study. Forty-five ambulant patients with COVID-19 (World Health Organization (WHO) disease severity 1 and 2 according to the WHO clinical ordinal scale), 21 hospitalized patients (WHO 3-4) with moderate COVID-19 and 79 patients with severe COVID-19 who required ventilation (WHO 5-7, 52 of which fulfilled acute respiratory distress syndrome criteria according to the Berlin definition of this condition ${ }^{41}$ ) were enrolled in this study. All patients with COVID-19 were tested positive for SARS-CoV-2 RNA via nasopharyngeal swabs. Twenty patients who presented with influenza-like symptoms but were tested negative for SARS-CoV-2 (FLI) and a total of 96 healthy donors who did not present any clinical sign of viral infection were enrolled as controls. The clinical characteristics of all participants are summarized in Supplementary Tables 1-3. For autopsy, informed consent was given by the next of kin, and autopsies were performed on the legal basis of section 1 SRegG BE of the autopsy act of Berlin and section 25(4) of the German Infection Protection Act. The sequencing of the post-mortem tissue was approved by the Ethics Committee of the Charité (EA2/066/20, EA1/144/13 and EA1/075/19 to H.R.) and by the Charité-BIH COVID-19 research board and complied with the Declaration of Helsinki. Additional use of anonymized clinical data is covered by section 25 of the Berlin Hospital Law and did not require further ethical or legal clearance.

\section{Isolation of PBMCs and serum}

Peripheral blood was drawn from each donor into EDTA collection tubes and, for selected samples (CD107a assay), into heparin tubes (BD Biosciences). PBMCs were separated from peripheral blood by Pancoll human (PAN-Biotech) density gradient centrifugation at room temperature. Cells were either used directly for analysis or stored in heat-inactivated fetal bovine serum (FCS; Pan-Biotech, P30-3602) with $10 \%$ dimethylsulfoxide at $-80^{\circ} \mathrm{C}$ before analysis. Serum samples were drawn from each donor into Vacutainer SSTTM tubes (BD Biosciences), centrifuged for $10 \mathrm{~min}$ at $2,000 \mathrm{~g}$ and stored at $-20^{\circ} \mathrm{C}$ before analysis.

\section{Flow cytometry analysis}

PBMCs were incubated with Fc blocking reagent (Miltenyi Biotec) according to the manufacturer's instructions. To exclude dead cells, the cells were stained using a Live/Dead (LD) Fixable Aqua Dead Cell staining kit (ThermoFisher, L34965). For surface antigen staining, the cells were incubated with monoclonal anti-human antibodies (Supplementary Table 4) for $20 \mathrm{~min}$ at $4{ }^{\circ} \mathrm{C}$. The Foxp3 Transcription Factor Staining Buffer Set (eBioscience, 00-5523-00) was applied before intracellular staining of transcription factors, cytotoxic molecules and cytokines. The samples were analysed using a FACS Fortessa X20 (BD Biosciences). Data were analysed using FlowJo Software v.10.3 (Treestar). Mean fluorescent intensity (MFI) values of NK cell populations were normalized to the MFI of lineage-negative marker-negative cells ${ }^{42}$. For intracellular cytokine staining of IFN $\gamma$ (BioLegend, 502527) and TNF (BioLegend, 502908), NK cells were stimulated for $4 \mathrm{~h}$ at $37^{\circ} \mathrm{C}$ in the presence of brefeldin A (Sigma-Aldrich) with phorbol 12-myristate 13-acetate (25 ng ml${ }^{-1}$, Sigma-Aldrich) and ionomycin (500 ng ml${ }^{-1}$, Sigma-Aldrich). Alternatively, NK cells were co-cultured with K562 target cells (American Type Culture Collection (ATCC) CCL-243, verified by ATCC) for $4 \mathrm{~h}$ at $37^{\circ} \mathrm{C}$ in the presence of brefeldin A. In Supplementary Table 4 , all antibodies are listed and assigned to staining panels A, B, C, D and E. Patient information and time point after onset of symptoms when the peripheral blood was obtained for each flow cytometry analysis are listed Supplementary Tables 1-3.

\section{In vitro culture of sorted NK cells}

Frozen PBMCs were gradually thawed at $37{ }^{\circ} \mathrm{C}$ and resuspended in RPMI medium (Gibco, 31870074) supplemented with $20 \%$ heat-inactivated FCS (Pan-Biotech P30-3602), L-glutamine $(200 \mathrm{mM}$, Gibco, 25030081), penicillin-streptomycin $\left(10,000 \mathrm{U} \mathrm{ml}^{-1}\right.$, Gibco, 5140122) and gentamicin (Lonza BioWhittaker, BW17-519L). Live cells were discriminated using a Live/Dead Fixable Aqua Dead Cell staining kit (ThermoFisher, L34965) and incubated in human Fc blocking reagent (Miltenyi Biotec) according to the manufacturer's instructions. The cells were stained with the following anti-human antibodies for 20 min at $4{ }^{\circ} \mathrm{C}$ : CD3 (eBioscience, 11-0039-42), CD4 (eBioscience, 11-0048-42), CD14 (BioLegend, 325604), CD19 (BioLegend, 363008), CD45 (BioLegend, 393409), CD7 (BioLegend, 343119) and CD56 (BioLegend, 362511). NK cells were sorted as $\mathrm{LD}^{-} \mathrm{Lin}^{-}$(CD3, CD4, $\mathrm{CD} 14$ and $\mathrm{CD} 19) \mathrm{CD} 45^{+} \mathrm{CD}^{+} \mathrm{CD} 56^{+}$using a FACS Aria II Cell Sorter (BD Biosciences).

Sorted NK cells were cultured in RPMI containing rh-IL-12 (20 ng ml${ }^{-1}$; PeproTech, 200-12H), rh-IL-15 (20 ng ml$^{-1}$; PeproTech, 200-15), rh-TGF $\beta$ (10 $\mathrm{ng} \mathrm{ml}^{-1}$; PeproTech, 100-21), rh-IFN $\alpha\left(10,000 \mathrm{U} \mathrm{ml}^{-1}\right.$; R\&D Systems, 11100-1) or rh-IFN $\beta$ (20 ng ml; R\&D Systems, MAB1835-SP) and cultured for 2-4 days at $37^{\circ} \mathrm{C}$ with $5 \% \mathrm{CO}_{2}$ as indicated.

\section{Chromium release assay}

A chromium release assay was performed as previously described ${ }^{43}$. In brief, full PBMCs were cultured overnight in RPMI supplemented with rh-IL-12 and rh-IL-15. For selected analyses, rh-TGF $\beta$ was added. NK cell frequencies in the PBMC fraction were determined using flow cytometry. $\mathrm{K} 562$ target cells were radioactively labelled by incubating $2 \times 10^{6} \mathrm{~K} 562$ cells in $450 \mu \mathrm{l}$ RPMI with $50 \mu \mathrm{l}$ of ${ }^{51} \mathrm{Cr}$ (CR-RA-8, Cr51, $185 \mathrm{MBq}, 5 \mathrm{mCi} \mathrm{ml}^{-1}$ ) for $2 \mathrm{~h}$ at $37^{\circ} \mathrm{C}$ on a rotator. After labelling, target cells were washed twice with RPMI before adding PBMCs at the indicated NK cell:target cell ratios (9:1,3:1,1:1 or 1:3). Following a co-culture incubation of $4 \mathrm{~h}\left(37^{\circ} \mathrm{C}\right.$ with $\left.5 \% \mathrm{CO}_{2}\right)$, the supernatant was collected and the ${ }^{51} \mathrm{Cr}$ released was quantified using a Wallac Wizard 1470 gamma counter. To quantify the maximal ${ }^{51} \mathrm{Cr}$ release for each experiment, the gamma count of the supernatant of target cells cultured without effector cells (spontaneous ${ }^{51} \mathrm{Cr}$ release) was subtracted from the gamma signal of labelled target cells only. The percentage of specific lysis or the percentage of ${ }^{51} \mathrm{Cr}$ release induced by NK cells from healthy donors in a 3:1 NK cell:target cell ratio (per cent of maximum ${ }^{51} \mathrm{Cr}$ release) was calculated for each sample.

\section{Degranulation assay of NK cells}

CD107a expression on NK cells of the PBMC fraction was measured as previously described ${ }^{44}$ (protocol 2 of the consensus protocol). In brief, PBMCs were cultured at $2 \times 10^{6} \mathrm{ml}^{-1}$ in RPMI overnight. NK cells were subsequently co-cultured for $2 \mathrm{~h}$ with $\mathrm{K} 562$ cells and stained for CD107a (eBioscience, 11-1079-42), CD56 (Beckman Coulter, A82943), CD8 (Beckman Coulter, IM2469), CD3 (Beckman Coulter, A94680) and CD45 (Beckman Coulter, B36294). The samples were analysed on a Navios-EX FACS (Beckman Coulter). Data were analysed using the Navios 2.0 software. CD107a mobilization of sorted NK cells (as described in the section 'In vitro culture of sorted NK cells') cultured in RPMI, supplemented with rh-IL-12, rh-IL-15 and rh-TGF $\beta$ as indicated, was analysed after co-culture with $\mathrm{K} 562$ cells at a 2:1 NK cell:target cell ratio for $4 \mathrm{~h}$ at $37^{\circ} \mathrm{C}$. Subsequently, cells were re-stained using a Live/ Dead Fixable Aqua Dead Cell staining kit, human Fc blocking reagent and the following antibodies: CD3 (eBioscience, 11-0039-42), CD4 (eBioscience, 11-0048-42), CD14 (BioLegend, 325604), CD19 (BioLegend, 363008), CD45 (BioLegend, 393409), CD7 (BioLegend, 343119), CD107a (BD Bioscience, 562622) and CD56 (BioLegend, 362511). The frequency of $\mathrm{CD} 107$ a expression was determined in $\mathrm{LD}^{-} \mathrm{Lin}^{-}$(CD3, CD4, $\mathrm{CD} 14$ and $\mathrm{CD} 19) \mathrm{CD} 45^{+} \mathrm{CD}^{+} \mathrm{CD} 56^{+} \mathrm{NK}$ cells using a FACS Fortessa $\mathrm{X} 20$ (BD Biosciences). 


\section{Conjugation assay}

NK cell:target cell adhesion was assessed as previously described ${ }^{45}$. In brief, PBMCs were separated from peripheral blood by a density gradient as described above. Cells were enriched by negative selection using a NK cell isolation kit (Miltenyi Biotec) according to the manufacturer's instructions. After isolation, NK cells were labelled for 20 min using a Celltrace Far Red Cell Proliferation kit (Invitrogen, C34564) according to the manufacturer's instructions. K562 target cells were labelled for $10 \mathrm{~min}$ at $37^{\circ} \mathrm{C}$ with cell proliferation dye (Invitrogen, 65-0842-85). After labelling, cells were washed with RPMI, mixed in a 1:4 NK cell: target cell ratio and centrifuged at $300 \mathrm{~g}$ for $1 \mathrm{~s}$. Cells were co-cultured for $1 \mathrm{~h}$ at $37^{\circ} \mathrm{C}$ before conjugates were quantified by flow cytometry.

\section{Co-culture of NK cells with SARS-CoV-2-infected cells}

PBMCs were separated from freshly drawn peripheral blood by a density gradient as described above. Cells were enriched by negative selection using a NK cell isolation kit (Miltenyi Biotec) according to the manufacturer's instructions. Enrichment was ensured by flow cytometry. After isolation, NK cells were stimulated for $24-48 \mathrm{~h}$ in RPMI 1640 supplemented with rh-IL-12 (PeproTech, 200-12H), rh-IL-15 (PeproTech, 200-15) and rh-TGF $\beta$ (PeproTech, 100-21) as indicated at $37^{\circ} \mathrm{C}$ with $5 \% \mathrm{CO}_{2}$. NK cells were washed with DMEM and co-cultured with SARS-CoV-2-infected Vero E6 cells (Cercopithecus aethiops kidney epithelial cells; ATCC CRL-1586, verified by ATCC) or Calu-3 cells (human bronchial epithelial cells; ATCC HTB-55, verified by ATCC) as indicated. Approximately 175,000 Vero E6 cells and 300,000 Calu-3 cells per well were seeded in 24-well plates $24 \mathrm{~h}$ before infection as indicated.

For masking experiments, NK cells were incubated with the neutralizing antibodies anti-NKp30 (clone F252), anti-NKp44 (clone KS38), anti-NKp46 (clone KL247), anti-NKG2D (clone BAT221 and clone ON72), anti-DNAM-1 (clone F5) and anti-2B4 (clone CO54) at room temperature 45 min before co-culture as indicated. For all blocking experiments, target cells were incubated with anti-HLA-I (clone A6/136). Monoclonal antibodies were provided by E. Marcenaro (University of Genova, Italy).

Cells were infected with the SARS-CoV-2/München 984 virus isolate (B.1 lineage, hCoV-19/Germany/BY-ChVir-984/2020, accession ID: EPI_ISL_406862; Pango lineage v.3.1.1, lineages v.2021-06-15) or the variant B.1.351 (hCoV-19/Germany/BW-ChVir22131/2021, accession ID: EPI_ISL_862149) at cell culture passage 2 (ref. ${ }^{46}$ ) with a multiplicity of infection (MOI) of 0.001 (Vero E6) or a MOI of 0.1 (Calu-3). Virus was diluted in OptiPro serum-free medium (Thermo Fisher). For infection, the supernatant was removed, cells were rinsed once with $0.5 \mathrm{ml}$ PBS (Thermo Fisher) and $200 \mu \mathrm{l}$ of virus-containing dilution was inoculated on the cells for $1 \mathrm{~h}$ at $37^{\circ} \mathrm{C}$. Next, $500 \mu \mathrm{l}$ of NK cell suspension was added $12 \mathrm{~h}$ after infection as indicated. Adherent cells were collected $12 \mathrm{~h}$ (Vero E6) or $24 \mathrm{~h}$ (Calu-3) after NK cell addition for isolation of viral RNA. For isolation of viral RNA, $35 \mu$ l of MagNA Pure 96 external lysis buffer (Roche) was added to the adherent cells. All samples were heat-inactivated for $10 \mathrm{~min}$ at $70^{\circ} \mathrm{C}$. Isolation and purification of viral RNA was performed using a MagNA Pure 96 System (Roche) according to the manufacturer's recommendations. Viral RNA was quantified using real-time PCR with reverse transcription (RT-PCR; E gene assay) as previously described ${ }^{47}$. All infection experiments were done under biosafety level 3 conditions with enhanced respiratory personal protection equipment.

\section{Viral load data analyses}

Viral load (RNA copies per swab) measurements were obtained based on a calibrated curve of viral RNA copies and RT-PCR cycle threshold values as previously described ${ }^{48}$. For the examination of temporal viral load dynamics, patients with at least two viral load measurements and two immune cell count measurements were included, and a linear regression was calculated. A maximum offset for both parameters was set to 7 days, and only infections with a duration of less than 40 days were considered. Regression analysis was performed using the seaborn (regplot) package v.0.11.1 and the scipy (stats.linregress) package (v.1.6.0) running under Python 3.9.1. For indicated analyses, patients were categorized as having either low or normal absolute immune cell counts according to whether their first immune cell count had a value below or above a threshold value. The following thresholds were used: 40 NK cells per $\mu \mathrm{l} ; 40 \mathrm{~B}$ cells per $\mu \mathrm{l} ; 360$ T cells per $\mu \mathrm{l} ; 200 \mathrm{CD}^{+} \mathrm{T}$ cells per $\mu \mathrm{l} ; 300 \mathrm{CD}^{+}$T cells per $\mu \mathrm{l} ; 600$ lymphocytes (CD45 ${ }^{+} \mathrm{CD} 14^{-}$) per $\mu \mathrm{l}$. Analysis was performed on hospitalized patients who tested positive (through SARS-CoV-2RT-PCR), including patients who tested positive in an intensive care unit ward at any point during their infection and including patients with severe COVID-19 of the study cohort (Extended Data Fig. 1, Supplementary Table 3).

\section{NK cell isolation from peripheral blood for single-cell sequencing}

Frozen PBMCs from 13 patients with severe COVID-19 (52 samples), 11 ambulant patients with COVID-19 (11 samples) from day 2 to day 68 after onset of symptoms (Supplementary Tables 1-3) and 5 healthy donors (5 samples) were thawed in RPMI (Gibco, 31870074) supplemented with $20 \%$ heat-inactivated FCS and incubated with Fc blocking reagent (Miltenyi Biotec) following the manufacturer's instructions. Up to $1 \times 10^{7}$ cells per $100 \mu$ l were stained with the following anti-human antibodies: CD3 (Miltenyi Biotec, 130-113-133), CD14 (Miltenyi Biotec, 130-113-152), CD19 (Miltenyi Biotec, 130-113-172), CD45 (BioLegend, 304008) and CD56 (Miltenyi Biotec, 130-113-305). To allow cell pooling, each sample was also incubated with one of eight different TotalSeq-C anti-human Hashtags (LNH-94;2M2, Barcoded, BioLegend, 394661, 394663, 394665, 394667, 394669, 394671, 394673 and 394675).4,6-diamidino-2-phenylindole(DAPI) was added before sorting to enable exclusion of dead cells. NK cells were identified and sorted as $\mathrm{DAPI}^{-} \mathrm{CD} 3^{-} \mathrm{CD} 14^{-} \mathrm{CD} 19^{-} \mathrm{CD} 45^{+} \mathrm{CD} 56^{+}$. All sortings used a MA900 Multi-Application Cell Sorter (Sony Biotechnology). Cell counting was performed using a MACSQuant flow cytometer (Miltenyi Biotec). Sorted NK cells were further processed for scRNA-seq.

For the generation of a NK cell-specific TGF $\beta$-response dataset, live cells in thawed PBMCs were discriminated using a Live/Dead Fixable Aqua Dead Cell staining kit (ThermoFisher, L34965) and incubated in human Fc blocking reagent (Miltenyi Biotec) according to the manufacturer's instruction. The cells were stained with the following anti-human antibodies for 20 min at $4{ }^{\circ} \mathrm{C}$ : CD3 (eBioscience, 11-0039-42), CD4 (eBioscience, 11-0048-42), CD14 (BioLegend, 325604), CD19 (BioLegend, 363008), CD45 (BioLegend, 393409), CD7 (BioLegend, 343119) and CD56 (BioLegend, 362511). NK cells were sorted as LD Lin $^{-}$(CD3, CD4, CD14 and CD19) $\mathrm{CD}^{+} 5^{+} \mathrm{CD} 7^{+} \mathrm{CD} 56^{+}$using a FACS Aria II Cell Sorter (BD Biosciences). Sorted NK cells were cultured in RPMI containing rh-IL-12 and rh-IL-15 with or without additional rh-TGF $\beta$ for 4 days at $37^{\circ} \mathrm{C}$ with $5 \% \mathrm{CO}_{2}$ before scRNA-seq.

\section{scRNA library preparation and sequencing}

The 10X Genomics workflow for cell capturing and scRNA gene expression (GEX) was applied to sorted NK cells using a Chromium Single Cell 5' Library \& Gel Bead kit and a Single Cell 5' Feature Barcode Library kit (10X Genomics). Final GEX was obtained after fragmentation, adapter ligation and final index PCR using the Single Index Kit T Set A. A Qubit HS DNA assay kit (Life Technologies) was used for library quantification, and fragment sizes were determined using the Fragment Analyzer with the HS NGS Fragment kit (1-6,000 bp; Agilent). Sequencing was performed on a NextSeq500 device (Illumina) using High Output v2 kits ( 150 cycles) with the recommended sequencing conditions for $5^{\prime}$ GEX libraries (read 1:26 nt, read 2: $98 \mathrm{nt}$, index 1: $8 \mathrm{nt}$ ).

\section{Single-cell transcriptome analysis}

Chromium single-cell data were processed using cellranger-3.1.0. The mkfastq and the count pipeline were used in default 
parameter settings for demultiplexing, alignment of reads to the Refdata-cellranger-hg19-1.2.0 genome, barcode and unique molecular identifier (UMI) counting, and calling of intact cells. The number of expected cells was set to 3,000. Further analysis was performed using the Seurat R-package (v.3.1.1 ${ }^{49}$. The pooled samples were separated using eight cite-seq hashtags (TotalSeq-C, BioLegend). Cells with more than $30 \%$ cite-seq reads for a particular hashtag were assumed as positively stained. Cells with no (undefined origin) or ambivalent assignments (doublets) were removed from further analysis. The resulting transcriptome profiles of NK cells from healthy donors, ambulant patients with COVID-19 and patients with severe COVID-19 were normalized and integrated as previously described ${ }^{50}$. Variable genes were detected and scaled. Data were scaled and UMAP was performed in default parameter settings using Scale Data, RunPCA and RunUMAP with 30 principal components. Quality control was performed by visual inspection of the fraction of mitochondrial genes, the number of detected genes and UMI counts per cell. No notable abnormalities were observed. Transcriptionally similar cells were clustered using shared nearest neighbour (SNN) modularity optimization with a SNN resolution of 0.2. Marker genes for clusters were identified using FindAllGenes with a log fold-change of $>2.5$ and a minimum of 0.1 expressing cells. The genes used for manual annotation of the function of the clusters was informed by previous publications ${ }^{16,20,51}$. One out of six clusters revealed B-cell-specific and monocyte-specific genes (CD19, CD14) and was excluded from further analysis.

Pseudotime trajectory analysis was performed using the monocle $2 \mathrm{R}$ package. Cells from each group (healthy, ambulant and severe COVID19) were randomly downsampled to an equal depth of 3,871 cells. The overall 11,613 cells were used for the calculation in monocle2. The top 1,000 differentially expressed genes across the five clusters (ranked by lowest $q$-value) were used to order the cells. The BEAM method was used to identify genes that significantly differed in expression level across a branch point ${ }^{22}$.

Single-cell data derived from NK cells cultured in presence and absence of TGF $\beta$ were analysed using the analog workflow except for clustering and trajectory analysis steps. The TGF $\beta$ response signature was defined on the basis of differentially expressed genes between samples with and without TGF $\beta$ treatment. To compare samples, two-sided Mann-Whitney $U$-test was used on log-normalized counts. Differentially expressed genes were identified (adjusted $P \leq 0.05$ and a $\log$ fold-change of $>2.2$ for enrichment and $<2.2$ for depletion). The heatmap of differentially expressed genes is based on $z$-transformed means of log-normalized counts. Hierarchical clustering was performed using Euclidian distances and WARD linkage criterium. All generated sequencing data were deposited into the NCBI Gene Expression Omnibus (accession number GSE184329).

\section{Analysis of peripheral blood NK cells from a publicly available dataset}

NK cell data were extracted from a publicly available PBMC single-cell sequencing dataset (GSE149689) consisting of 4 samples from healthy participants, 11 samples from patients with COVID-19 and 5 samples from patients with severe influenza ${ }^{30}$. In the first step, the individual samples and 59,572 cells were integrated and subdivided into 10 clusters in a UMAP representation with a SNN resolution of 0.1. A heatmap of cell-type-defining genes was used to identify 10,604 NK cells from the PBMCs. GSEA for TGF $\beta$-induced and TGF $\beta$-suppressed NK cell genes was performed as described below.

\section{Analysis of non-haematopoietic and haematopoietic cell populations from lung tissue}

The publicly available lung single-nucleus dataset (European GenomePhenome Archive reference ID: EGAS00001004689) consisting of three patients who died from pneumonia unrelated to COVID-19 (as controls) and seven patients who died from COVID-19-associated pneumonia ${ }^{31}$ was aligned to a hg19 reference transcriptome that included the SARS-CoV-2 genome (NCBI reference sequence ID: NC_045512) using cellranger v3.0.1 (10X Genomics). Ambient RNA removal was performed with SoupX $(\mathrm{v} 1.4 .5)^{52}$. First, the 10 samples and 53,709 cells were integrated as previously described ${ }^{50}$ for the NK cell single-cell sequencing dataset and subdivided into 33 clusters in a UMAP representation with a SNN resolution of 1 . A heatmap of 13 genes was used to identify NK cells from the lung, and cluster 25 was defined as the NK-cell-specific cluster of 845 cells. The remaining cell populations were identified according to Gassen et al. ${ }^{31}$.

\section{GSEA}

GSEA was performed for each cell on the basis of differences in log-normalized counts to the mean of all cells analysed using 1,000 randomizations (false discovery rate $<0.5$ and normalized $P<0.2$ ) $^{53}$. For visualization, the normalized enrichment score per cell within the indicated gene set (upregulation or downregulation) was plotted. Gene sets (Hallmark, Reactome and KEGG) were obtained from the Molecular Signatures Database (MSigDB, v.6.2) ${ }^{54,55}$. The NK-cell-specific TGF $\beta$ response gene set was newly generated in this study. The GSEA-enrichment plot (score curve) of TGF $\beta$-suppressed genes in lung-tissue-resident NK cells was performed on pre-ranked differences between medians of expressing cells in otherwise default parameter settings. Only genes expressed in at least one group were considered. The median was set to 0 if no expressing cells were found.

\section{In vitro NK cell exposure to patient serum}

Sorted NK cells from healthy donors were cultured for $48 \mathrm{~h}$ in RPMI containing IL-12, IL-15 and IL-2 (25 $\mathrm{ng} \mathrm{ml}^{-1}$; PeproTech, 212-12) as indicated and $20 \%$ of either serum from another healthy donor (Supplementary Table 1) or from a patient with severe COVID-19 (Supplementary Table 3). Unless stated otherwise, IL-12, IL-15 and serum were used. For indicated experiments, the patient sera were pre-incubated with antibodies directed against TGF $\beta 1$, TGF $\beta 2$ and TGF $\beta 3$ (R\&D Systems, MAB1835-SP), anti-IL-6 (5 $\mathrm{g} \mathrm{m} \mathrm{ml}^{-1}$; R\&D Systems, MAB206), anti-IL-10 (30 $\mu \mathrm{g} \mathrm{ml}$; R\&D Systems, MAB217) or anti-IL-15 (5 $\mu \mathrm{g} \mathrm{ml}$; eBioscience, 16-0157-82) as indicated for $10 \mathrm{~min}$ before being added to the culture and using identical culture conditions. After $48 \mathrm{~h}$, protein expression levels of T-bet were measured, the frequency of CD107a expression in NK cells was analysed after $4 \mathrm{~h}$ of co-culture with K562 cells using flow cytometry or viral replication was determined, as described above.

\section{Cytokine measurements}

Cytokine levels were measured using a bead-based multiplex cytokine array (Human Cytokine 25-Plex ProcartaPlex Panel 1B, Thermo Fisher Scientific). Before the assay, serum samples were diluted 1:3 in dilution buffer provided with the kit. TGF $\beta$ was detected using a Human TGF $\beta 1$ Simplex ProcartaPlex kit (Thermo Fisher Scientific). Before measuring serum TGF $\beta 1$, the bioactive form of TGF $\beta 1$ was generated by incubating the serum with $1 \mathrm{~N} \mathrm{HCl}$ followed by neutralization with $1.2 \mathrm{~N} \mathrm{NaOH}$ according to the manufacturer's instructions. The samples were incubated with antibody-coated magnetic beads for $30 \mathrm{~min}$ at room temperature with shaking, then incubated overnight at $4{ }^{\circ} \mathrm{C}$ followed by a 1 -h incubation period at room temperature. All subsequent incubation steps were performed according to the manufacturer's instructions. The assay plates were read using a Luminex MAGPIX system and quantified using XPONENT analysis software (Luminex). IFN $\alpha$ serum concentration was analysed using a Simoa IFN $\alpha$ Advantage kit (Quanterix) according to the manufacturer's instructions.

\section{Statistical analysis and reproducibility}

All statistical tests were performed with Graph Pad Prism V7 software as indicated for each analysis $\left({ }^{*} P \leq 0.05,{ }^{* *} P \leq 0.01,{ }^{* * *} P \leq 0.001\right.$ and ${ }^{* * * *} P \leq 0.0001$; NS, not significant). Representative data of at least three independent experiments are shown in Figs. 1d, e, g, $2 \mathrm{f}$ and $4 \mathrm{c}$, f, and 
Extended Data Figs. 15a, b, c, d. Representative data of two independent experiments are shown in Extended Data Fig. 15j, k, n, o.

\section{Reporting summary}

Further information on research design is available in the Nature Research Reporting Summary linked to this paper.

\section{Data availability}

All generated sequencing data were deposited at NCBI Gene Expression Omnibus (accession number GSE184329).

41. Force, A. D. T. et al. Acute respiratory distress syndrome: the Berlin Definition. JAMA $\mathbf{3 0 7}$ 2526-2533 (2012)

42. Cossarizza, A. et al. Guidelines for the use of flow cytometry and cell sorting in immunological studies (second edition). Eur. J. Immunol. 49, 1457-1973 (2019).

43. Verneris, M. R., Karimi, M., Baker, J., Jayaswal, A. \& Negrin, R. S. Role of NKG2D signaling in the cytotoxicity of activated and expanded CD8 ${ }^{+}$T cells. Blood 103, 3065-3072 (2004).

44. Bryceson, Y. T. et al. A prospective evaluation of degranulation assays in the rapid diagnosis of familial hemophagocytic syndromes. Blood 119, 2754-2763 (2012).

45. Deguine, J., Breart, B., Lemaitre, F., Di Santo, J. P. \& Bousso, P. Intravital imaging reveals distinct dynamics for natural killer and $\mathrm{CD} 8^{+} \mathrm{T}$ cells during tumor regression. Immunity 33, 632-644 (2010).

46. Wolfel, R. et al. Virological assessment of hospitalized patients with COVID-2019. Nature 581, 465-469 (2020).

47. Corman, V. M. et al. Detection of 2019 novel coronavirus (2019-nCoV) by real-time RT-PCR. Euro Surveill. 25, 2000045 (2020).

48. Jones, T. C. et al. Estimating infectiousness throughout SARS-CoV-2 infection course. Science 373, eabi5273 (2021).

49. Butler, A., Hoffman, P., Smibert, P., Papalexi, E. \& Satija, R. Integrating single-cell transcriptomic data across different conditions, technologies, and species. Nat. Biotechnol. 36, 411-420 (2018).

50. Stuart, T. et al. Comprehensive integration of single-cell data. Cell 177, 1888-1902.e21 (2019).

51. Smith, S. L. et al. Diversity of peripheral blood human NK cells identified by single-cell RNA sequencing. Blood Adv. 4, 1388-1406 (2020).

52. Young, M. D. \& Behjati, S. SoupX removes ambient RNA contamination from droplet-based single-cell RNA sequencing data. Gigascience 9, giaa151 (2020).

53. Subramanian, A et al. Gene set enrichment analysis: a knowledge-based approach for interpreting genome-wide expression profiles. Proc. Natl Acad. Sci. USA 102, 15545-15550 (2005)

54. Li, S. et al. Molecular signatures of antibody responses derived from a systems biology study of five human vaccines. Nat. Immunol. 15, 195-204 (2014).

55. Liberzon, A. et al. Molecular signatures database (MSigDB) 3.O. Bioinformatics 27 $1739-1740$ (2011).

56. Sivori, S. et al. Human NK cells: surface receptors, inhibitory checkpoints, and translational applications. Cell. Mol. Immunol. 16, 430-441 (2019).

Acknowledgements The authors are most grateful to the patients for their consent in participating in this study. We thank members of the Diefenbach laboratory, the Mashreghi laboratory, the Kruglov laboratory, the Klose laboratory and the Romagnani laboratory for valuable discussions on the manuscript. Masking antibodies against NKp3O (clone F252) and DNAM-1 (clone F5) were provided by D. Pende (IRCCS Ospedale Policlinico San Martino, 16132 Genoa, Italy) and NKp46 (clone KL247) and NKp44 (KS38) by S. Parolini (Sezione di Oncologia e Immunologia Sperimentale, Dipartimento di Medicina Molecolare e Traslazionale, Università di Brescia). We are grateful to the staff at the Benjamin Franklin Flow Cytometry (BFFC) Facility (M. Fernandes and A. Branco) and the DRFZ flow cytometry facility (J. Kirsch, A. C. Teichmuller and T. Kaiser) for support in cell sorting. The BFFC is supported by DFG Instrument grants INST 335/597-1 FUGG und INST 335/777-1 FUGG. We are indebted to K. Oberle, F. Egelhofer, J. Heinze and A. Sebastiampillai for technical assistance and advice. We thank A. Bayindir, F. Cicek and E. Daka for support in the management of patient material. This work was supported by the following entities: Deutsche Forschungsgemeinschaft (TR-SFB 84/AO2 and A06, TR-SFB241/ A01, SPP1937-DI764/7 to A.D.; TRR130/P16 and P17 to A.R. and H.R.; TR-SFB 84/AO7 to C.D.; TRR241/AO4 to A.K.; RA 2491/1-1 to H.R.; SFB-TR 84/B08, SFB 1449/ZO2 to M.A.M); the European Research Council (ERC-2010-AdG 268978 to A.R.); the Einstein Foundation Berlin (Einstein Professorship to A.D. and M.A.M.); the state of Berlin and the "European Regional Development Fund" to M.-F.M. (ERDF 2014-2020, EFRE 1.8/11, Deutsches Rheuma-Forschungszentrum); the Berlin Institute of Health with the Starting Grant Multi-Omics Characterization of SARS-CoV-2 infection, Project 6 "Identifying immunological targets in COVID-19" to A.D. and M.-F.M.: the Russian Ministry of Science and Higher Education of the Russian Federation (grant 075-152019-1660) and the Russian Foundation for Basic Research (no. 17-00-00268 (https://kias.rfbr. ru/index.php)) to A.K; the Leibniz Association (Leibniz Collaborative Excellence, TargArt) to T.K. and M.-F.M.; the German Federal Ministry of Education and Research (BMBF) projects "NaFoUniMedCovid19" (FKZ: 01KX2O21)-COVIM (AP-4) to V.M.C., L.E.S. and A.D.; RECAST (01KI20337) to B.S., V.M.C., L.E.S., J. Roehmel. and M.A.M.; 82DZL009B1 to M.A.M.; Fondazione AIRC per la Ricerca sul Cancro to E.M. T.C.J. is in part funded through the NIAID-NIH CEIRS contract HHSN272201400008C. C.U.D. acknowledges support by a Rahel Hirsch Habilitationsstipendium. The autopsies were facilitated by the Biobank of the Department of Neuropathology, Charité-Universitätsmedizin Berlin and supported by the BMBF (Organostrat, Defeat Pandemics)

Author contributions M.W. and C.T. performed most of the experiments and analysed the data. M.F.-G. performed the flow cytometry-based sorting of NK cells and library preparation for scRNA sequencing. D.N. contributed to the experiments with SARS-CoV-2-infected cells. T.C.J. performed the viral load data analyses. T.H., P.N., S.M., I.M., U.K., C.M., C.D., A.K. and G.A.H., helped with experiments. F.H. and P.D. analysed the RNA-seq data. S.F. and M.M. performed the serum cytokine measurements. S.A., S.T., S.Z., E.V., F.K., L.E.S., L.H., M.A.M., V.M.C., R.L.C., T.A., B.S., J. Roehmel., T.S., C.C., H.R., J. Radke, T.K., V.M., K.A. and T.S. supported the management of patient samples and clinical data. J.A.T., E.M., I.M. and C.D. helped with the design of the in vitro experiments. M.W., C.T., M.F.-M. and A.R. contributed to the development of the study concept. A.D. and M.F.-M. designed, directed and supervised the study. A.D. wrote the manuscript with input from co-authors.

Competing interests The authors declare no competing interests.

Additional information

Supplementary information The online version contains supplementary material available at https://doi.org/10.1038/s41586-021-04142-6.

Correspondence and requests for materials should be addressed to Mario Witkowski or Andreas Diefenbach.

Peer review information Nature thanks the anonymous reviewers for their contribution to the peer review of this work. Peer review reports are available.

Reprints and permissions information is available at http://www.nature.com/reprints. 
a

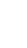

NK cells $>40 / \mu \mathrm{l}$
NK cells $\leqq 40 / \mu \mathrm{l}$

T cells $>360 / \mu \mathrm{l}$

T cells $\leqq 360 / \mu$ l

$\mathrm{CD}^{+}{ }^{\mathrm{T}}$ cells $>200 / \mu \mathrm{l}$

$\mathrm{CD}^{+} \mathrm{T}$ cells $\leqq 200 / \mu \mathrm{l}$

$\mathrm{CD}^{+} \mathrm{T}^{\mathrm{T}}$ cells $>300 / \mu \mathrm{l}$

$\mathrm{CD}^{+} \mathrm{T}$ cells $\leqq 300 / \mu \mathrm{l}$

B cells $>40 / \mu \mathrm{l}$

B cells $\leqq 40 / \mu l$

lymphocytes > 600/ul

lymphocytes $\leqq 600 / \mu$ l
$-0.22$

-0.12
-0.15

OVID-19 Hospitalized

mean median SD

$\begin{array}{lll}-0.14 & -0.12 & 0.32\end{array}$

$-0.14$

$-0.08$

$-0.10 \quad-0.12$

0.19

$\begin{array}{cc}.32 & 250 \\ .19 & 29\end{array}$

0.27

0.43

222

57

$\begin{array}{llll}-0.11 & -0.12 & 0.26 & 232\end{array}$

$\begin{array}{lll}-0.23 & -0.16 & 0.47\end{array}$

47

$\begin{array}{llll}-0.10 & -0.12 & 0.27 & 195\end{array}$

$\begin{array}{llll}-0.20 & -0.16 & 0.38 & 84\end{array}$

$\begin{array}{llll}-0.12 & -0.12 & 0.27 & 268 \\ -0.31 & -0.14 & 0.67 & 15\end{array}$

$\begin{array}{llll}-0.31 & -0.14 & 0.67 & 15\end{array}$

$0.27 \quad 215$

0.41
64

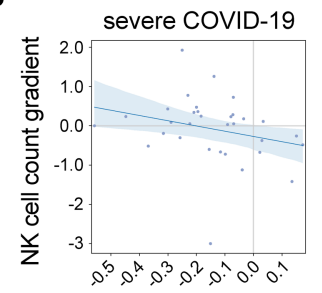

$\log _{10}$ viral load gradient

C

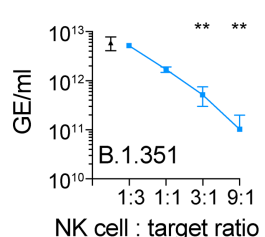

d

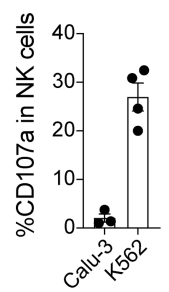

Extended Data Fig. 1 | The temporal viral load decline is related to the NK cell status. (a) Regressions for temporal viral load according to absolute peripheral blood immune cell count. The viral load trajectory was estimated by linear regression for COVID-19 patients with at least two viral load measurements each, and whose first absolute immune cell count was below or above the indicated threshold (see also methods). Analysis was performed on SARS-CoV-2 RNA-positive patients who were hospitalized, hospitalized and who tested positive on an ICU ward at any point during their infection (Intensive Care Unit group), and severe COVID-19 patients of the study cohort (Supplementary Table 3). The table lists the mean and median temporal viral load regression gradient, standard deviation (SD) and number of patients (n) per group. (b) The relationship between temporal gradient of $\log _{10}$ viral load and NK cell count change. For each of 32 severe COVID-19 patients with at least two viral load measurements and at least two NK cell count measurements, a linear regression was calculated for each series and a dot corresponding to the

COVID-19 Intensive Care Unit

Severe COVID-19 study cohort

mean median SD $\mathrm{n} \quad \mathrm{me}$

$\begin{array}{cccc}-0.13 & -0.12 & 0.31 & 183 \\ -0.07 & -0.07 & 0.18 & 23 \\ -0.09 & -0.11 & 0.22 & 160 \\ -0.24 & -0.16 & 0.47 & 46 \\ & & & \\ -0.10 & -0.12 & 0.22 & 169 \\ -0.24 & -0.15 & 0.52 & 37 \\ & & & \\ -0.10 & -0.12 & 0.21 & 140 \\ -0.19 & -0.14 & 0.42 & 66 \\ -0.12 & -0.12 & 0.24 & 197 \\ -0.31 & -0.10 & 0.80 & 10 \\ & & & \\ -0.1 & -0.12 & 0.22 & 153 \\ -0.22 & -0.15 & 0.44 & 53\end{array}$

$\begin{array}{llll}-0.22 & -0.15 & 0.44 & 53\end{array}$

mean

$\begin{array}{cccc}-0.24 & -0.16 & 0.51 & 42 \\ -0.05 & -0.09 & 0.12 & 5 \\ & & & \end{array}$

$\begin{array}{llll}-0.12 & -0.14 & 0.22 & 32\end{array}$

$\begin{array}{llll}-0.42 & -0.19 & 0.76 & 15\end{array}$

$\begin{array}{llll}-0.13 & -0.14 & 0.22 & 36\end{array}$

$\begin{array}{llll}-0.5 & -0.20 & 0.86 & 11\end{array}$

$\begin{array}{llll}-0.11 & -0.14 & 0.23 & 27\end{array}$

$\begin{array}{llll}-0.36 & -0.17 & 0.66 & 20\end{array}$

$\begin{array}{llll}-0.17 & -0.15 & 0.34 & 44\end{array}$

$\begin{array}{llll}-0.86 & -0.14 & 1.25 & 3\end{array}$

$\begin{array}{llll}-0.13 & -0.14 & 0.24 & 30 \\ -0.37 & -0.15 & 0.72 & 17\end{array}$

-




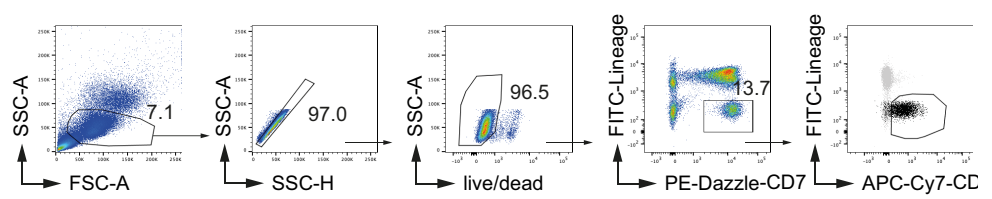

b

healthy
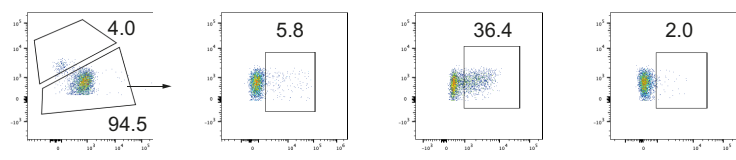

FLI
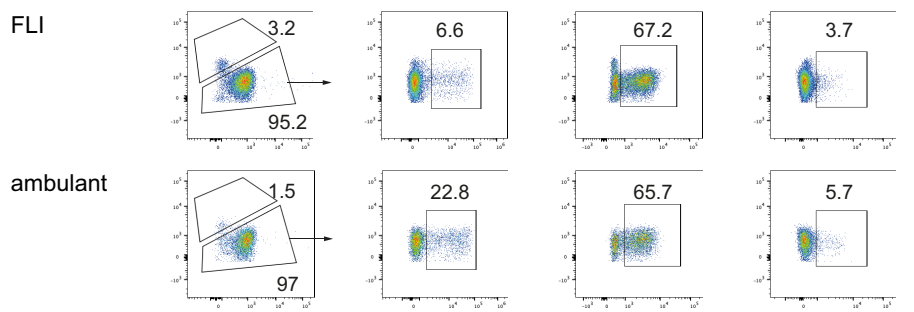

moderate
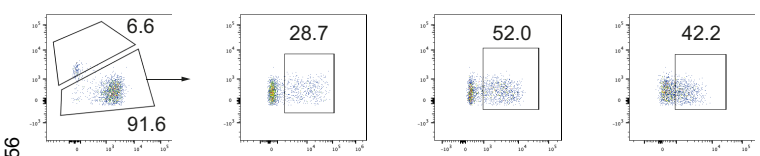

severe
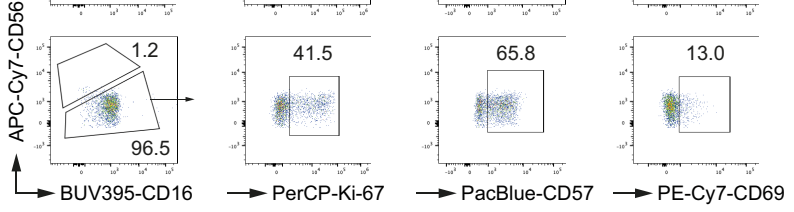

$\bigcirc$ healthy $\bigcirc \mathrm{FLI} \bigcirc$ ambulant $\bigcirc$ moderate $\bigcirc$ severe

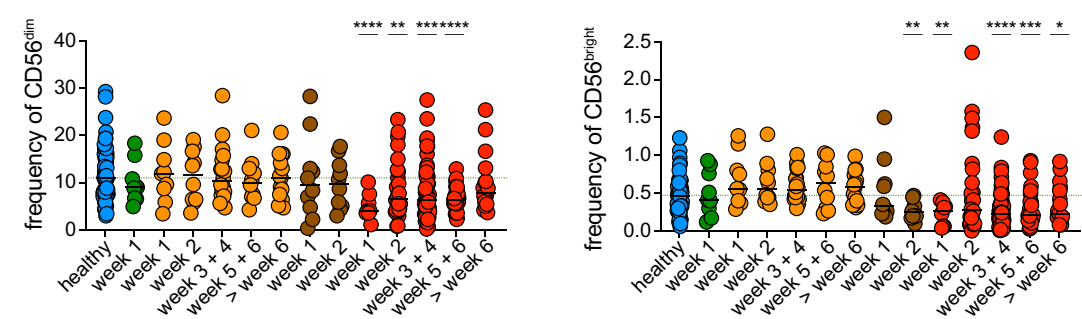

d

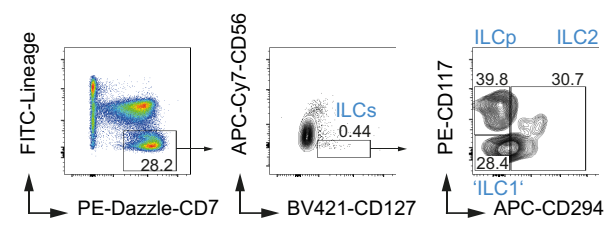

e

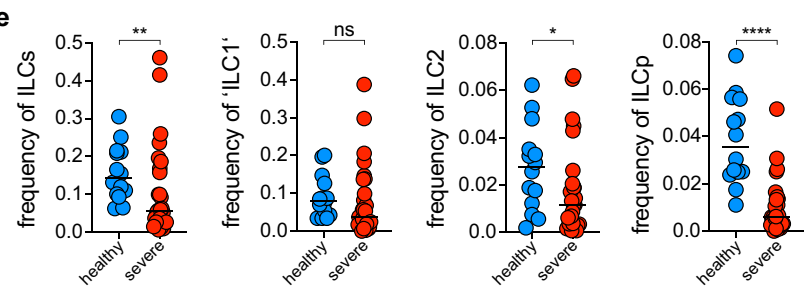

Extended Data Fig. 2 |See next page for caption. 


\section{Article}

Extended Data Fig. 2 | Reduction of peripheral blood NK cells and ILC1-like cells during COVID-19. (a) Gating strategy for the identification of CD56 ${ }^{\text {bright }}$ and CD56 ${ }^{\text {dim }}$ peripheral blood NK cells. (b) Continuation of the gating depicted in (a) and representative flow cytometry plots for the indicated markers in CD56 ${ }^{\text {dim }} \mathrm{NK}$ cells of a healthy donor, patient with flu-like illness (FLI), ambulant patient with COVID-19, patient with moderate COVID-19 and a patient with severe COVID-19 (b). (c) Frequency of CD56 ${ }^{\text {dim }}$ and CD56 ${ }^{\text {bright }}$ NK cells (median) in the peripheral blood of healthy donors and patients. Independent measurements of 53 healthy donors ( $n=53$ ), 9 flu-like illness (FLI, $n=9$ ), 29 ambulant COVID-19 $(n=62), 17$ moderate $(n=19)$ and 45 severe COVID-19 patients $(n=133)$ between week 1 and week 11 after onset of symptoms. Statistical analysis was performed using a One-way ANOVA followed by a two-sided Mann-Whitney $U$-test comparing healthy $v s$. FLI or COVID-19 groups. The dashed line indicates the median frequency in healthy donors. (d,e) Identification of ILC subsets in 14 healthy donors $(n=14)$ and 8 patients with severe COVID-19 $(n=28$ independent measurements). (d) Gating strategy for ILC with pre-gate set on live, CD45 single lymphocytes. (e) Frequencies (median) of indicated ILC subsets in both groups (independent measurements of two-sided Mann-Whitney $U$-test, ILCs; $\mathrm{p}=0.007,{ }^{\prime}$ ILC1'; $=0.09$, ILC2; $\mathrm{p}=0.04$, ILCp; $\left.\mathrm{p}<0.0001\right)\left({ }^{*} ; \mathrm{p} \leq 0.05,{ }^{* *} ; \mathrm{p} \leq 0.01\right.$, $\left.{ }^{* * *} ; \mathrm{p} \leq 0.001,{ }^{* * * *} ; \mathrm{p} \leq 0.0001\right)$. 
a

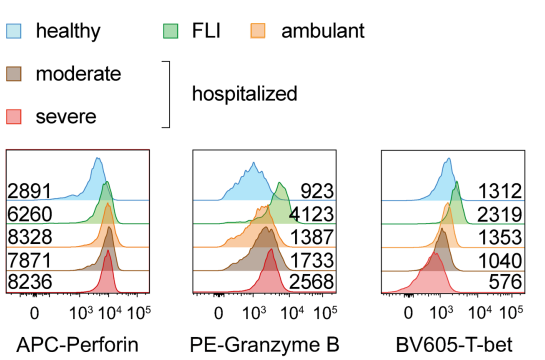

b

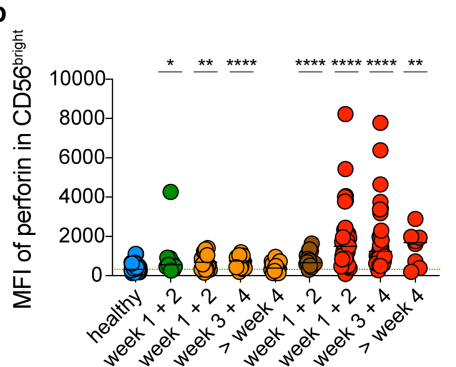

C

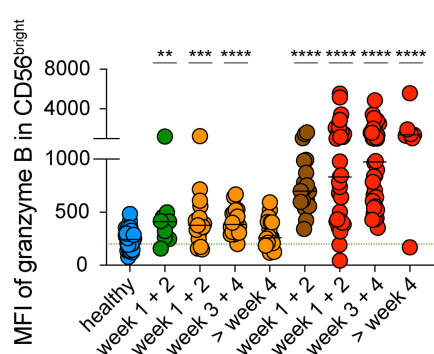

d

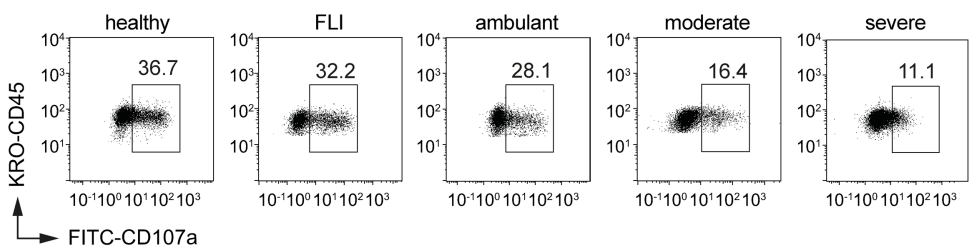

e

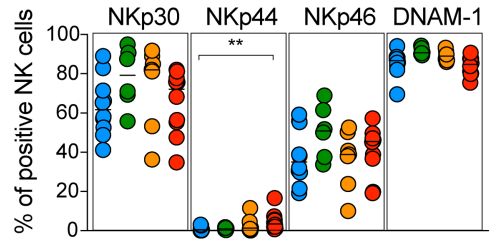

f

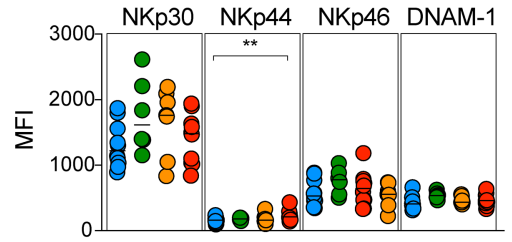

g

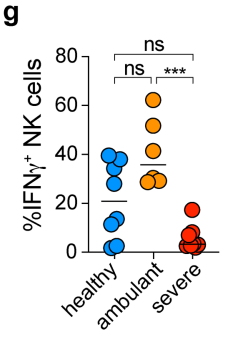

h

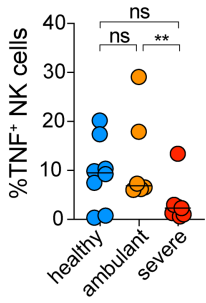

i

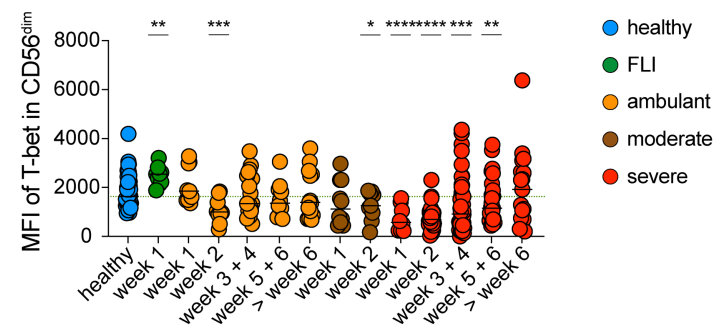

Extended Data Fig. 3 | See next page for caption. 


\section{Article}

Extended Data Fig. 3 | NK cells upregulate the expression of cytotoxic molecules but fail to express IFN $\gamma$ and TNF during severe COVID-19.

(a) Representative flow cytometry analysis showing the mean fluorescence intensity (MFI) of the indicated proteins in CD56 ${ }^{\mathrm{dim}} \mathrm{NK}$ cells from the indicated patient groups. (b,c) MFI (median) of perforin (b) and granzyme B (c) in CD56 ${ }^{\text {bright }}$ peripheral blood NK cells. Independent measurements from 44 ( $n=44$ for perforin) or 50 healthy donors $(n=50$ for granzyme $B), 9$ patients with $\operatorname{FLI}(n=9), 24$ ambulant COVID-19 ( $n=56), 17$ moderate COVID-19 ( $n=19)$ and 30 severe COVID-19 patients $(n=73)$ from week 1 to 6 after onset of symptoms. (d) Representative flow cytometry plots of cell surface CD107a expression by NK cells from indicated groups after $4 \mathrm{~h}$ co-culture with $\mathrm{K} 562$ cells. Pre-gate was set on CD3 CD56 lymphocytes. (e,f) Frequency (median) of expressing NK cells (e) and MFI (f) of indicated immunoreceptors on NK cells of 9 healthy donors ( $n=9$ independent measurements), 6 patients with FLI ( $n=6), 7$ ambulant $(n=7)$ and severe 9 COVID-19 $(n=10)$ (Frequency of NKp44 ${ }^{+} \mathrm{NK}$ cells healthy $u s$. severe $\mathrm{p}=0.004, \mathrm{MFI}$ of NKp $44^{+} \mathrm{NK}$ cells healthy $v$ s. severe $\left.\mathrm{p}=0.022\right)$. (g,h) PBMC of healthy donors $(n=8)$, ambulant patients with COVID-19 $(n=6)$ and patients with severe COVID-19 $(n=8)$ were stimulated with PMA/lonomycin and the frequency (median) of IFN $\gamma^{+}(\mathbf{g})$ and $\mathrm{TNF}^{+}(\mathbf{h}) \mathrm{NK}$ cells was determined by flow cytometry. Statistical analysis was performed using the two-sided Mann-Whitney $U$-test $\left(\mathrm{IFN} \gamma^{+} \mathrm{NK}\right.$ cells ambulant $v$ s. severe $\mathrm{p}=0.0007$; $\mathrm{TNF}^{+}$ $\mathrm{NK}$ cells ambulant $v s$. severe $\mathrm{p}=0.008$ ). (i) Mean fluorescence intensity (MFI, median) of T-bet in CD56 ${ }^{\mathrm{dim}} \mathrm{NK}$ cells across the disease course. Independent measurements of 53 healthy donors $(n=53)$, 9 patients with FLI $(n=9), 29$ ambulant $(n=62), 17$ moderate $(n=19)$ and 45 severe COVID-19 patients $(\mathbf{n}=133)$. For $(\mathbf{b}-\mathbf{c}, \mathbf{e}-\mathbf{f})$ and (i) statistical analysis was performed using a One-way ANOVA followed by a two-sided Mann-Whitney $U$-test comparing healthy $v s$. FLI or COVID-19 groups. The dashed line indicates the median MFI or frequency of healthy donors. ${ }^{*} ; \mathrm{p} \leq 0.05,{ }^{* *} ; \mathrm{p} \leq 0.01,{ }^{* * *} ; \mathrm{p} \leq 0.001$, ${ }^{* * * *} ; \mathrm{p} \leq 0.0001$ ). 


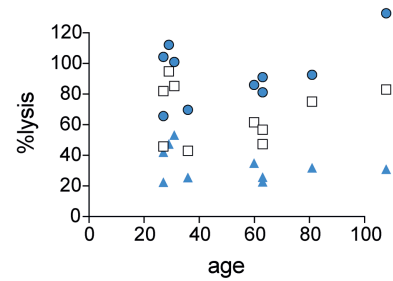

- $3: 1 \quad r=-0.143$

$p=0.715$

$\square 1: 1 \quad r=-0.185$

$\Delta 1: 3 \quad r=-0.202$ b

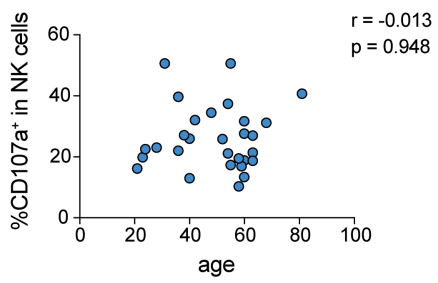

c
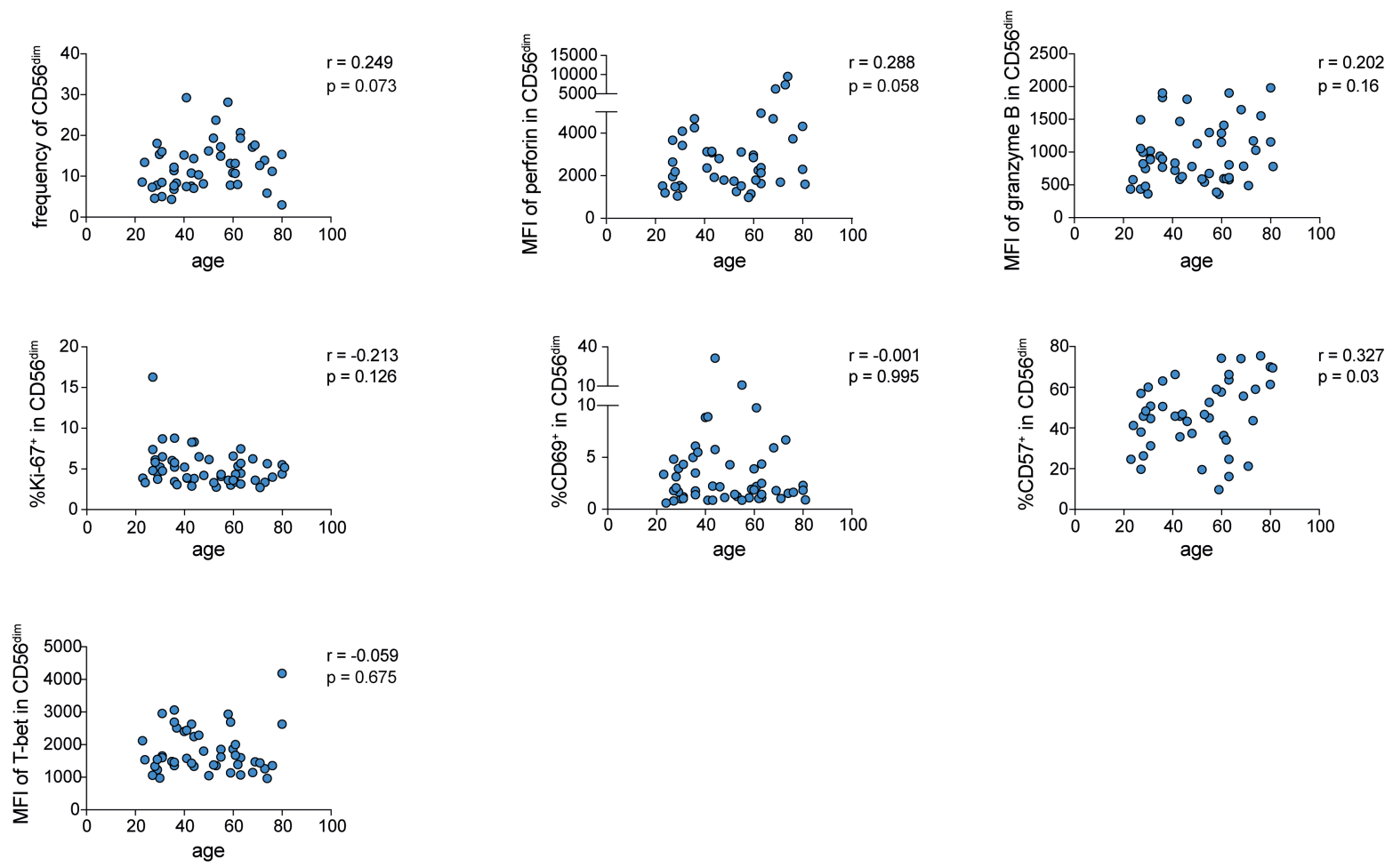

d
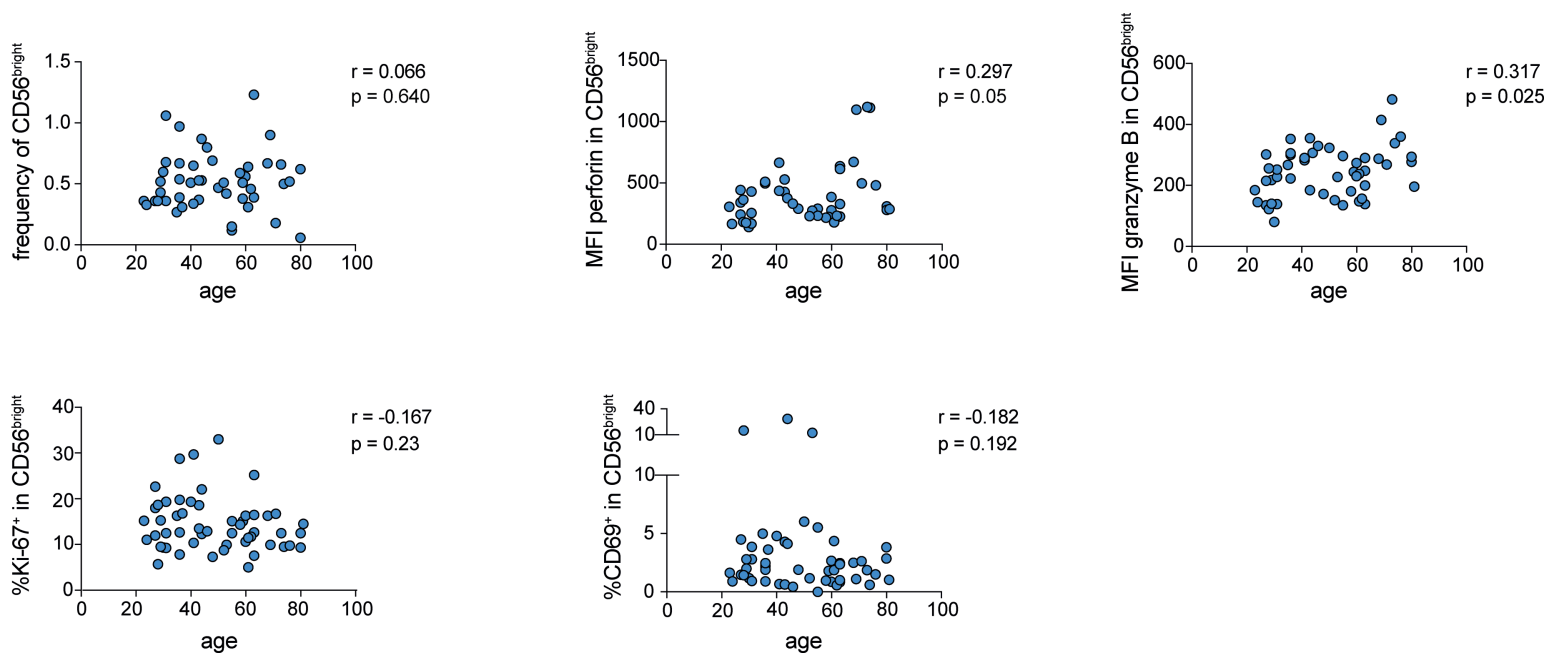

Extended Data Fig. 4 | Dysregulated NK cell function in severe COVID-19 does not correlate with age. (ard) Two-tailed spearman correlation of age and ${ }^{51} \mathrm{Cr}$ release in indicated NK cell: target cell ratios in a single experiment (a, $\mathrm{n}=9$ donors), CD107a expression in NK cells $(\mathbf{b}, \mathrm{n}=29)$ and expression of indicated markers measured by flow cytometry in CD 56 ${ }^{\mathrm{dim}}(\mathbf{c})$ and CD 56 ${ }^{\text {bright }}$ (d) NK cells of healthy donors $(n=44$ for perforin and CD $57, n=50$ for granzyme $B$, $\mathrm{n}=53$ for all others). Age in years is represented by the $\mathrm{x}$-axis whilst the indicated read out is represented by the $y$-axis. 

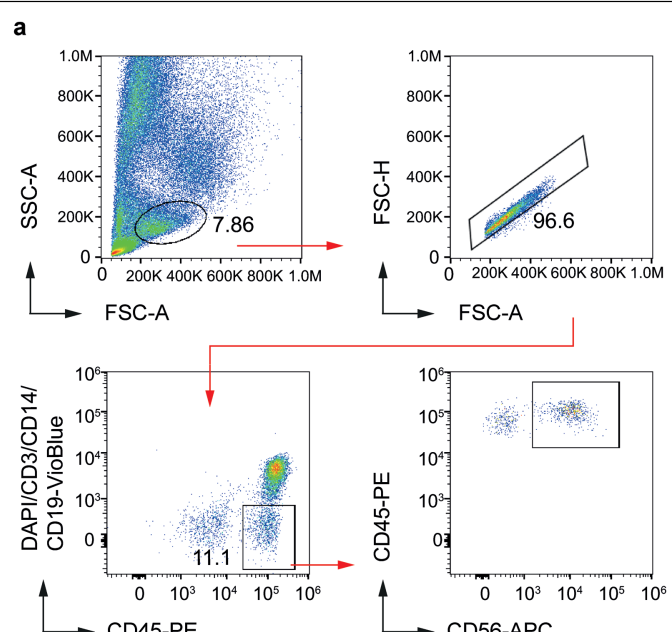

d

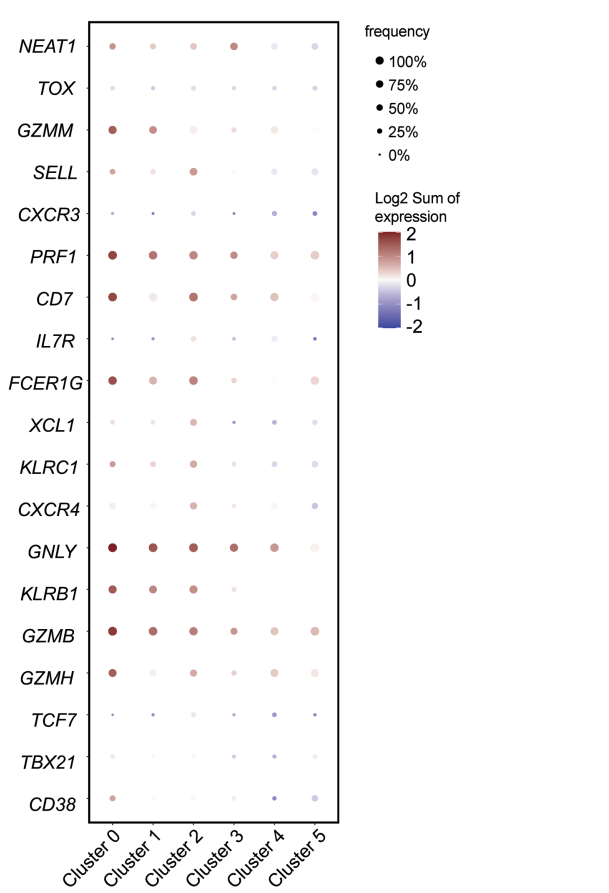

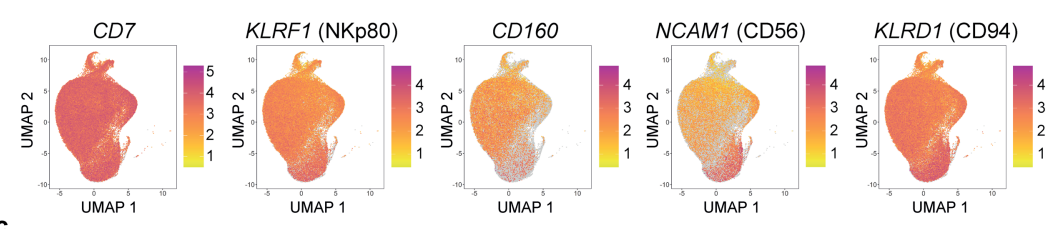
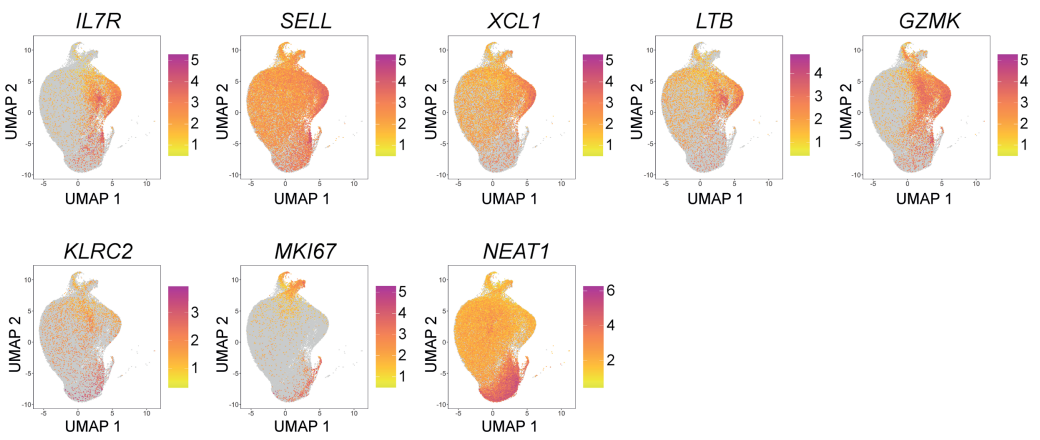

f
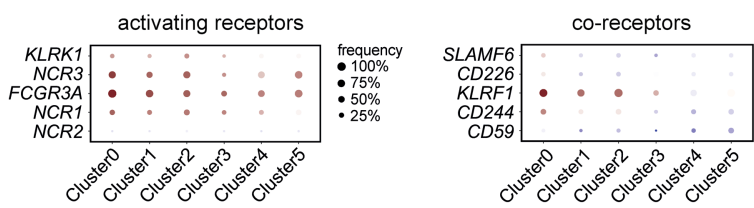

g Reactome cell cycle checkpoints
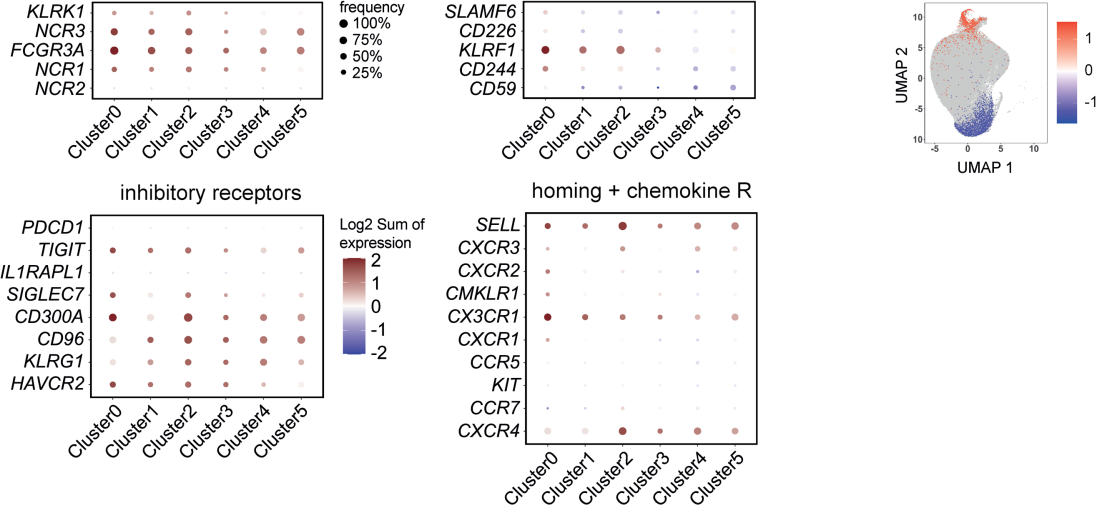

h

O healthy OFLI O ambulant O moderate O severe

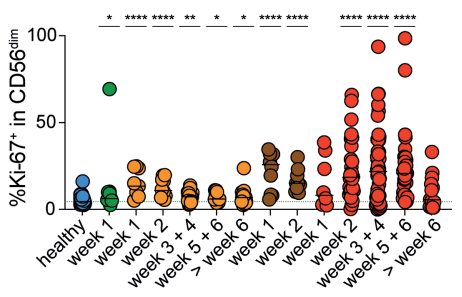

Extended Data Fig. 5 | Gene expression profile of individual NK cell clusters. (a) Gating strategy for FACS of peripheral blood NK cells subjected to single cell RNA sequencing. (b,c,e) UMAP representation depicting the expression levels of indicated genes. (d,f) Dot plots depicting the expression of the indicated genes in the NK cell clusters. Dot size represents the frequency of cells expressing the indicated gene. Selection of genes for each group in (f) according to Sivori et $\mathrm{al}^{56}$. (g) Single cell gene set enrichment analysis for the indicated gene set in all samples. Single cells with enriched gene expression are

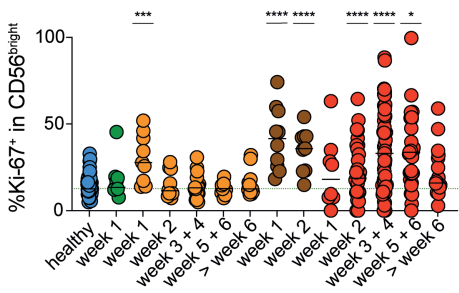

displayed as red dots, cells with depletion of the genes are displayed as blue dots. (h) Frequency of Ki-67 $7^{+}$D56 ${ }^{\text {dim }}$ and CD56 $6^{\text {bright }}$ NK cells (median). Independent measurements of 53 healthy donors $(n=53), 9$ flu-like illness (FLI, $n=9), 29$ ambulant $(n=62), 17$ moderate $(n=19)$ and 45 severe COVID-19 patients $(n=133)$ between week 1 and week 11 following onset of symptoms. Statistical analysis was performed using a One-way ANOVA followed by a two-sided Mann-Whitney $U$-test comparing healthy $v s$. FLI or COVID-19 groups. The dashed line indicates the median frequency of $\mathrm{Ki}-67^{+} \mathrm{NK}$ cells in healthy donors. 
a

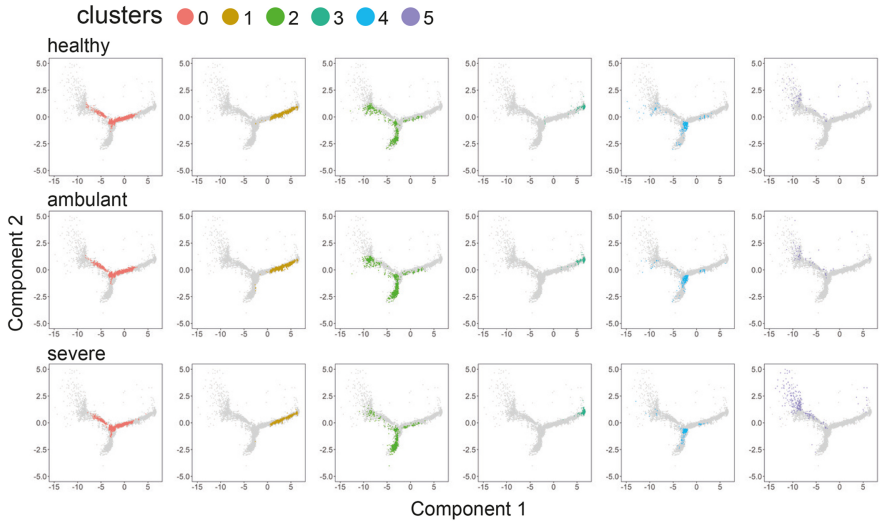

c

state $1 \bigcirc_{2} \bigcirc \bigcirc_{4} \bigcirc 5 \bigcirc 6 \quad d$

$97 \bigcirc 9911 \bigcirc 12$

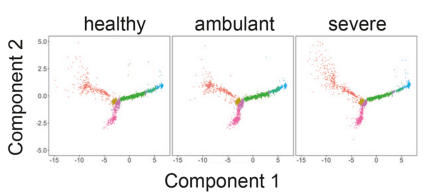

e
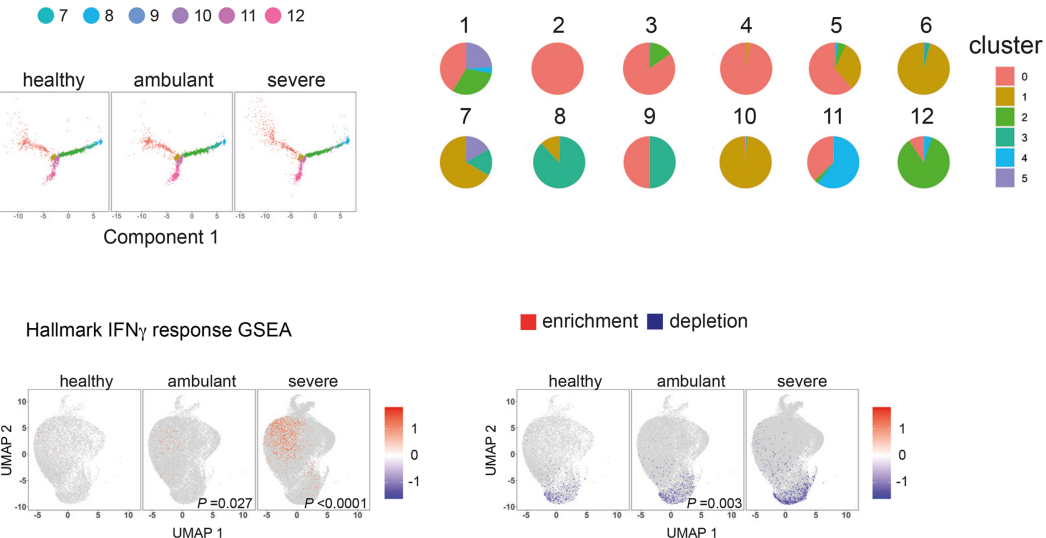

- enrichment $\mathbf{d e p l e t i o n}$

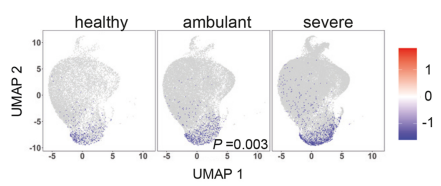

$f$
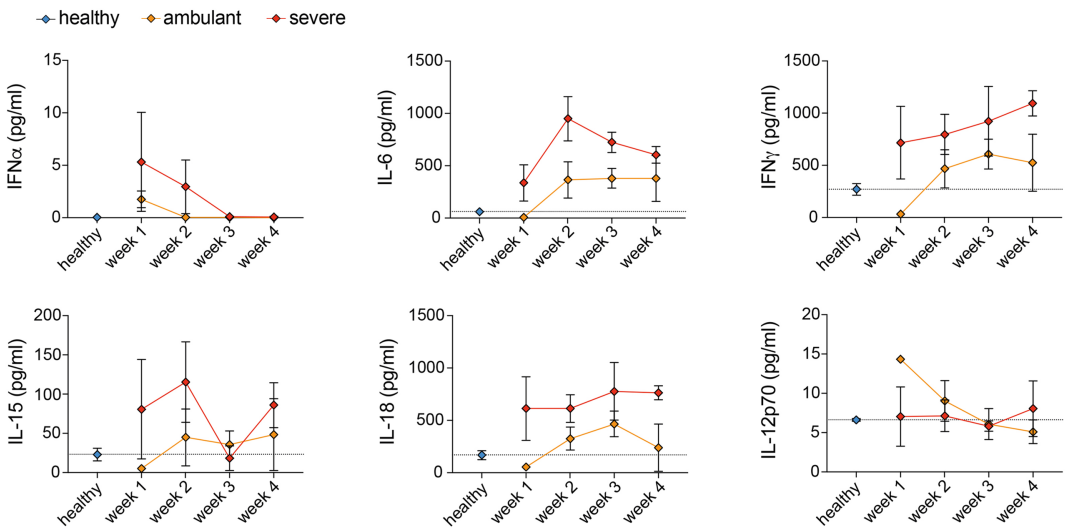

h

$$
\text { g }
$$
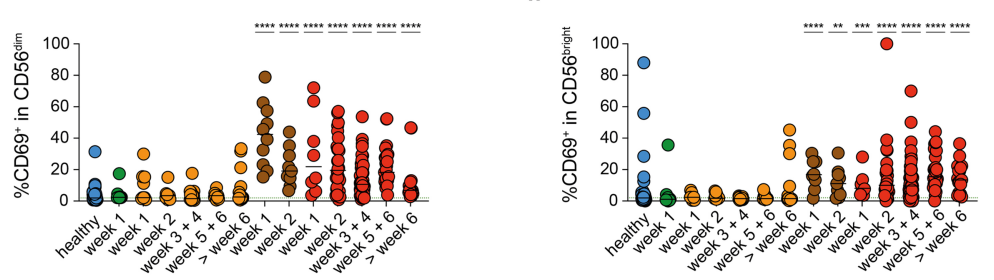

O healthy O FLI O ambulant o moderate o severe

Extended Data Fig. 6 | See next page for caption. b
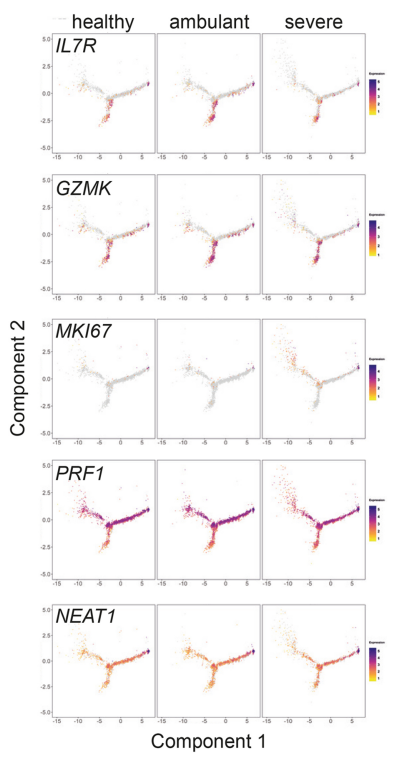
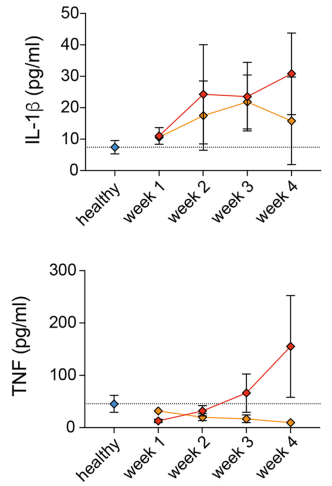


\section{Article}

Extended Data Fig. 6 | Differentiation trajectories of NK cells towards terminally differentiated NK cells. (a-d) Pseudotime trajectories of a total of 11,613 randomly selected NK cell transcriptomes from all groups. (a) Isolated visualization of each NK cell cluster in the pseudotime trajectory analysis. (b) Expression of indicated genes in the pseudotime trajectories. (c) Cell states in the trajectory analysis. (d) Pie charts depicting the representation of each NK cell cluster in the different cell states. (e) Single cell gene set enrichment analysis (GSEA) of the indicated gene set in the differentiated NK cell clusters (clusters 0,1 and 3 ) of all samples. Single cells with enriched gene expression are displayed as red dots and cells with depletion of the genes are displayed as blue dots. Significance of the enrichment or depletion was calculated using the two-sided Fisher's exact test by comparing the indicated group with the group left-sided (ambulant $v s$. healthy and severe $v$ s. ambulant, respectively). (f) Serum cytokine levels over the course of COVID-19. For IFN $\alpha$ data points represent independent measurements of 6 healthy donors $(n=6), 20$ ambulant patients with COVID-19 $(n=27)$ and 17 patients with severe COVID-19 $(n=26)$ at the indicated time points after onset of symptoms. Group size of 4 to 10 samples for COVID-19 patients per timepoint. For all other cytokines, data points represent independent measurements from 33 healthy donors $(n=33)$, 15 ambulant patients with COVID-19 $(n=20)$ and 6 patients with severe COVID-19 $(n=17)$ with a group size of 3 to 8 samples for COVID-19 patients. Patients receiving corticosteroid treatment were excluded from the analysis except for the IFN $\alpha$ serum measurements. Bars represent the mean \pm s.e.m. The dashed line indicates the mean serum concentration of the cytokine in healthy donors. Statistical analysis was performed using the two-sided Mann-Whitney $U$-test. (g,h) Frequency (median) of CD69 $9^{+} \mathrm{CD}^{\text {dim }}(\mathbf{g})$ and CD56 $6^{\text {bright }} \mathrm{NK}$ cells $(\mathbf{h})$. Independent measurements of 53 healthy donors $(n=53)$, 9 flu-like illness (FLI, $n=9), 29$ ambulant $(n=62), 17$ moderate $(n=19)$ and 45 severe COVID-19 patients ( $n=133$ ) between week 1 and week 11 after onset of symptoms. (i) Quantification of $\mathrm{CD} 57^{+} \mathrm{CD} 56^{\mathrm{dim}} \mathrm{NK}$ cells (median). Independent measurements from $44(n=44)$ healthy donors, 9 patients with FLI $(n=9)$, 24 ambulant $(n=56), 17$ moderate $(n=19)$ and 30 severe COVID-19 patients $(n=73)$ from week 1 to 8 after onset of symptoms. Statistical analysis in $\mathbf{g}-\mathbf{i}$ was performed using a One-way ANOVA followed by a two-sided Mann-Whitney $U$-test comparing healthy $v s$. FLI or COVID-19 groups. The dashed line indicates the median frequency in healthy donors. 
a

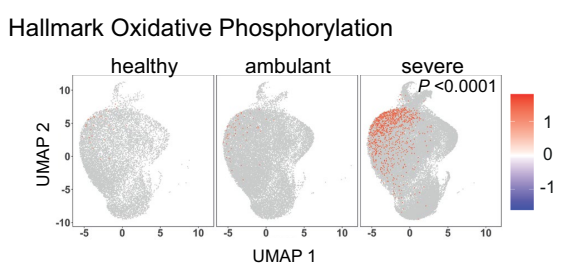

Reactome Metabolism of mRNA

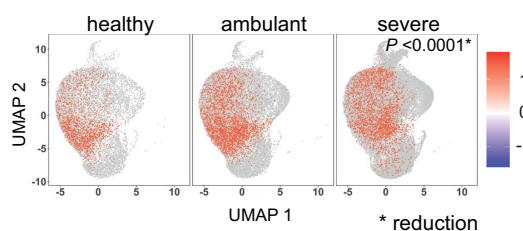

Reactome Metabolism of proteins

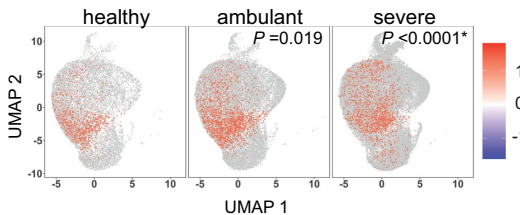

Reactome Translation

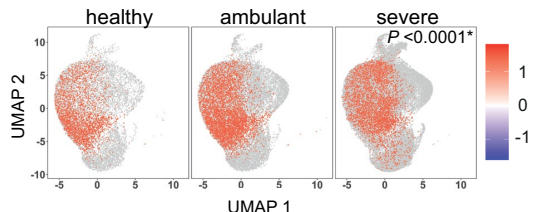

b

KEGG Intestinal Immune network for IgA production

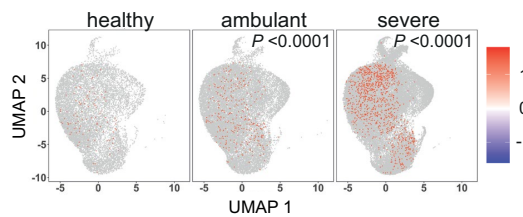

Extended Data Fig. 7 |NK cells show profound changes in gene networks related to cellular metabolism and intestinal IgA production during COVID-19. (a,b) Single cell gene set enrichment analysis (GSEA) of the indicated gene sets in the differentiated NK cell clusters (clusters 0,1 and 3 ) of all samples. Single cells with enriched gene expression are displayed as red dots enrichment

depletion
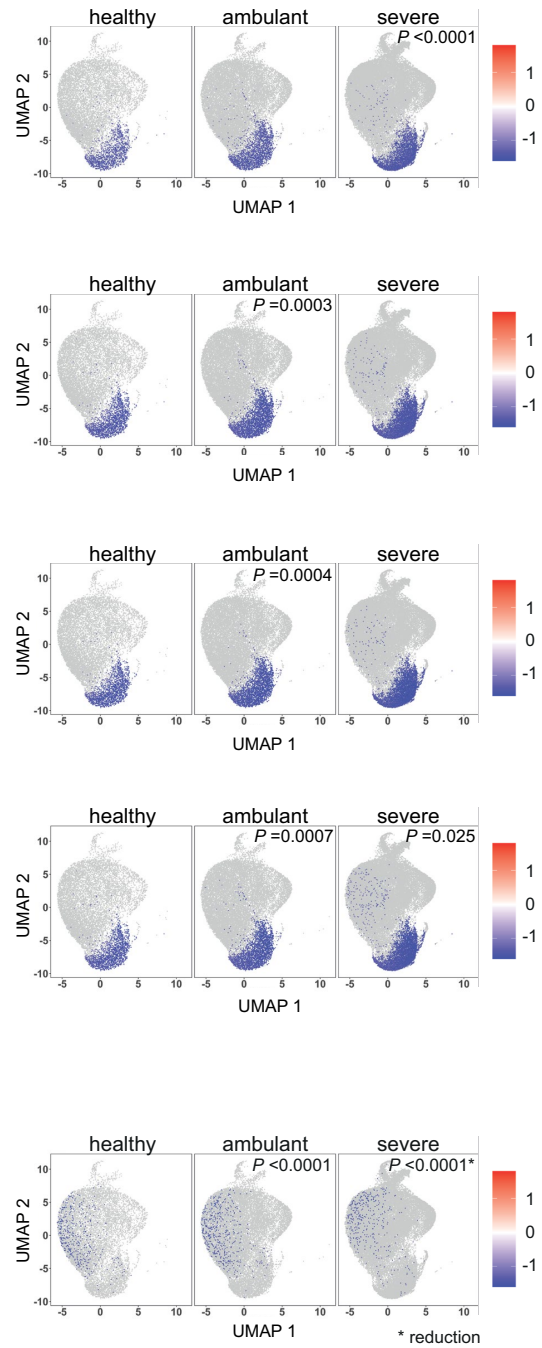

and cells with depletion of the genes are displayed as blue dots. Significance of the enrichment or depletion was calculated using the two-sided Fisher's exact test by comparing the indicated group with the group left-sided (ambulant $v s$. healthy and severe $v s$. ambulant, respectively). P-value* describes a reduction in enrichment or depletion. 
a

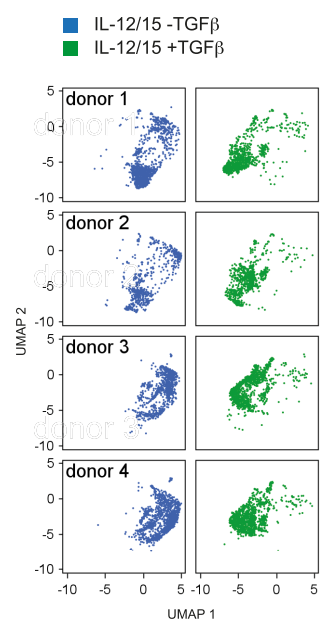

e

$\square$ healthy $\square$ ambulant $\quad \square$ severe
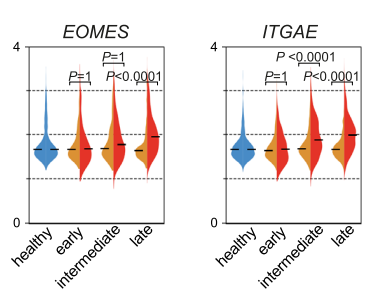

b

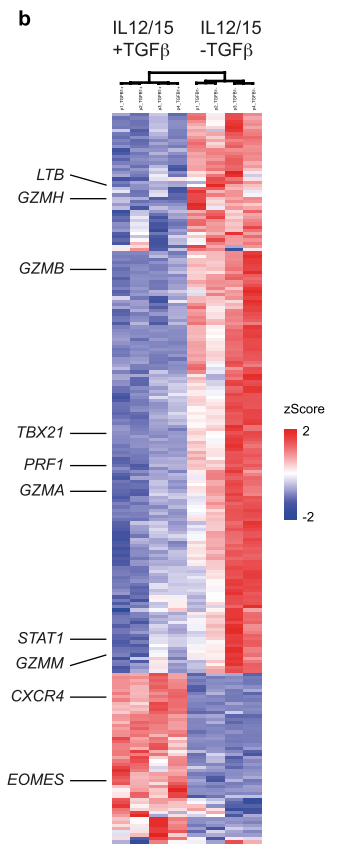

f

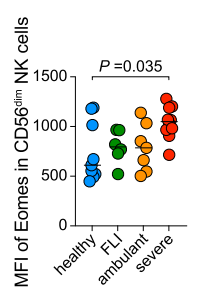

c

TGF $\beta$-induced

NK genes

${ }^{5} P<0.0001$

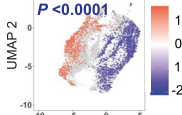

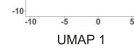

d

Hallmark PI3K AKT mTOR signaling Cell Adhesic

$\begin{array}{rl}{ }^{5} P & P<0.0001 \\ P & <0.0001\end{array}$

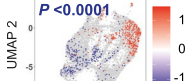

$\int_{-10}^{-10} \quad-5 \quad 0$

${ }^{5} P<0.0001$
$P<$

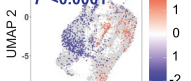

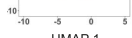

Adhesion Migration

Chemotaxis

${ }^{5} P<0.0001$

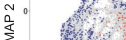

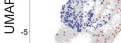

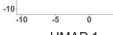

g

KEGG Cell Adhesion molecules

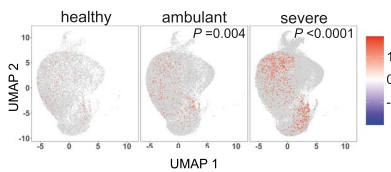

Hallmark PI3K AKT mTOR signaling
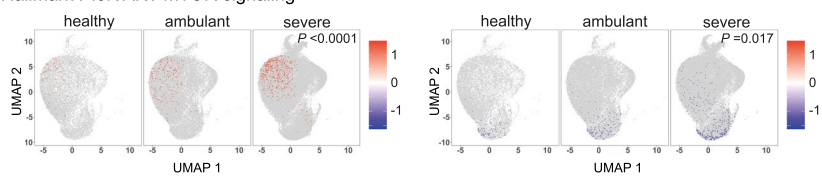

KEGG Natural Killer cell mediated cytotoxicity
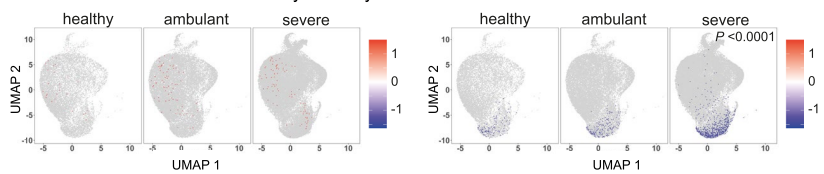

Reactome DAG and IP3 signaling
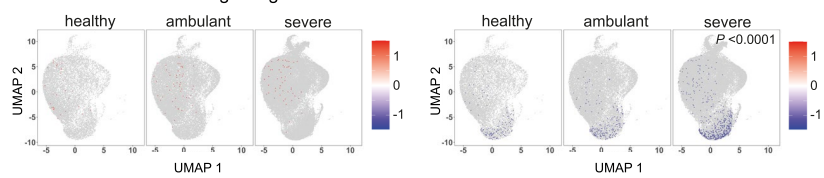

h

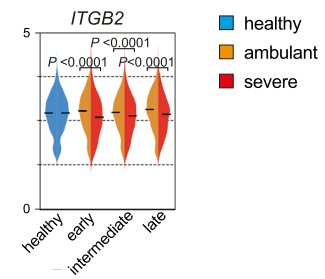

Extended Data Fig. $8 \mid$ See next page for caption. 
Extended Data Fig. 8 | Genes related to cell adhesion are suppressed in NK cells during severe COVID-19 and by invitro exposure to TGF $\beta$.

$(\mathbf{a}, \mathbf{b})$ Peripheral blood NK cells of 4 healthy donors were FACS-sorted and cultured in vitro in the presence of either IL-12, IL-15 and TGF $\beta$ or IL-12 and IL-15 alone and a total of 8137 single cell transcriptomes were generated. (a) UMAP representation of single cell transcriptomes of the four donors for both conditions. (b) Heatmap shows differentially expressed genes between both conditions. Upregulated genes are displayed in red, downregulated genes in blue. (c,d) Single cell GSEA of the NK cell-specific TGF $\beta$ response gene data set (c) or the indicated gene sets (d) projected on the UMAP of the scRNA-seq data obtained from NK cells cultured in vitro in the presence or absence of TGF $\beta$ as described above. Red dots represent cells with increased expression of the indicated gene set. Blue dots represent cells with a depletion of genes within the indicated gene set. Significance of the enrichment or depletion was calculated using the two-sided Fisher's exact test. (e,h) Violin Plot showing the median expression of the indicated genes in differentiated NK cell clusters
$(0,1$ and 3$)$ of healthy individuals and in ambulant and severe COVID-19 patients during the course of disease (Early: $\leq$ day 14 after symptom onset, intermediate: day 15-28, late: > day 28, two-sided Mann-Whitney $U$-test, p-value adjusted for multiple comparisons). (f) MFI of Eomes (median) was measured in CD56 $6^{\mathrm{dim}}$ NK cells from 9 healthy donors ( $n=9$ independent measurements), 6 patients with FLI ( $n=6), 7$ ambulant $(n=7)$ and 9 severe COVID-19 $(n=10)$. Statistical analysis was performed using a One-way ANOVA followed by a two-sided Mann-Whitney $U$-test comparing healthy $v$ s. FLI or COVID-19 groups. (g) Single cell gene set enrichment analysis (GSEA) of the indicated gene sets in the differentiated NK cell clusters (clusters 0,1 and 3 ) of all samples. Single cells with enriched gene expression are displayed as red dots and cells with depletion of the genes are displayed as blue dots. Significance of the enrichment or depletion was calculated using the two-sided Fisher's exact test by comparing the indicated group with the group left-sided (ambulant $v s$. healthy and severe vs. ambulant, respectively). 
a
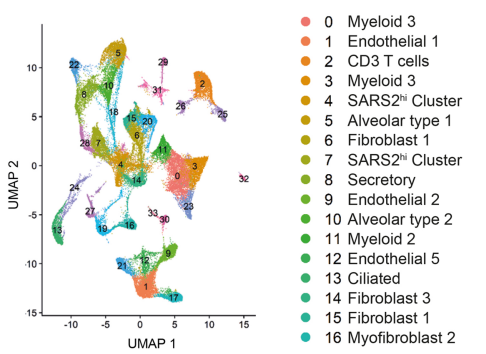

- 17 Endothelial 4

18 Differentiating alveolar type 2

20 Fyofribroblast 2

21 Endothelial 3

22 Basal
23 Myeloid 1

24 Ciliated diff

25 NK cells

26 B cells

27 Myofibroblast 3

28 Secretory diff

30 Lymphatic endothelial cells

31 Proliferating Lymphocytes

32 Mast cells

c

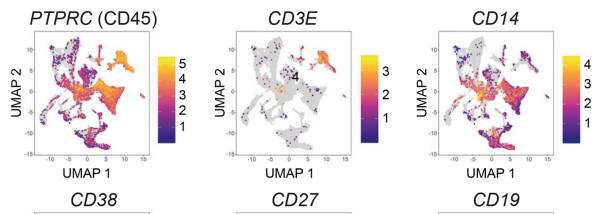

UMAP 1"
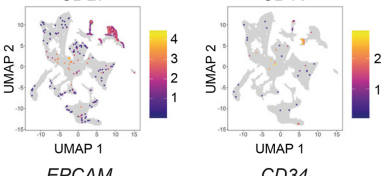

\begin{tabular}{|l|l|}
4 \\
UMAP 1
\end{tabular}
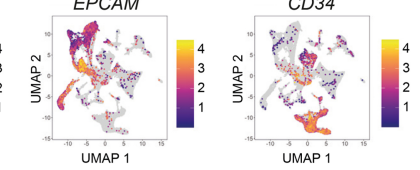

d

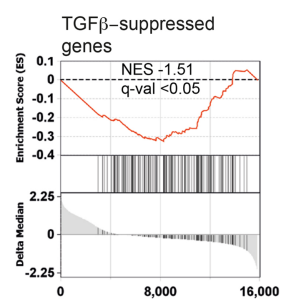

e
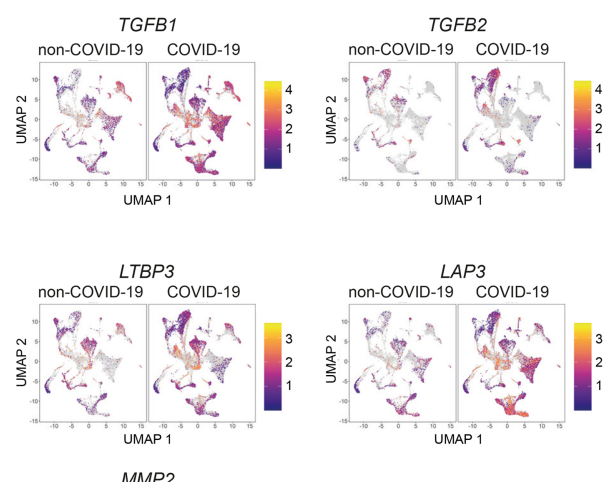

non-COVID-19 COVID-19

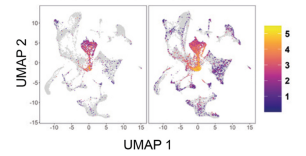

Extended Data Fig. 9|See next page for caption.
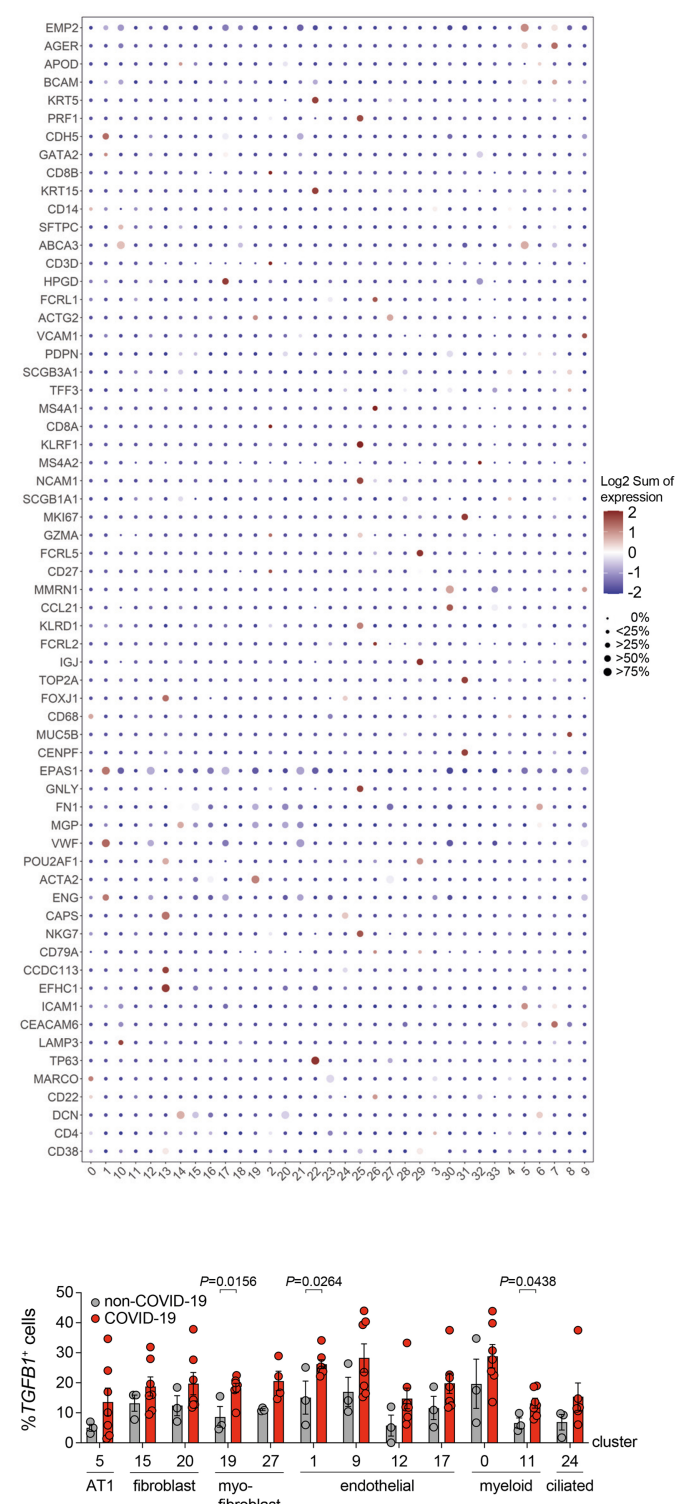

h TGFB1 $^{+}$
SARS-CoV-2-negative $\quad \begin{aligned} & \text { TGFB1+ } \\ & \text { SARS-CoV-2-positive }\end{aligned}$

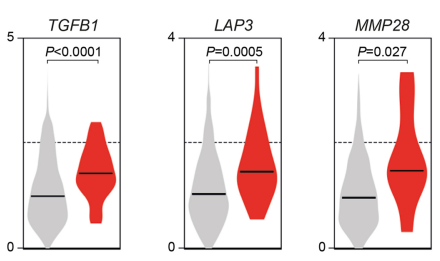


Extended Data Fig. 9 | TGF $\beta$ expression is induced in lung tissue hematopoietic and non-hematopoietic cell populations during COVID-19. (a-h) Single-nucleus sequencing of lung tissue from patients with SARS-CoV-2negative pneumonia (non-COVID-19) and severe COVID-19 ${ }^{31}$. (a) UMAP

visualization of single-nucleus transcriptomes $(>52,000)$ and identification of cellular populations according to $\left(\right.$ ref. $\left.^{31}\right)$. (b) Dot plot depicting the expression of the indicated genes in the various clusters. Dot size represents the frequency of cells expressing the indicated gene. (c) UMAPs showing the expression of the indicated genes in all cells. (d) GSEA of TGF $\beta$-suppressed NK cells genes in lung tissue-resident NK cells extracted from the data set. (e,f) UMAP representation of indicated genes in all cells of non-COVID-19 and COVID-19 patients.

(g) Quantification of the frequency of $T G F B 1^{+}$cells per cluster per patient in both groups. Only clusters represented by cells were included. Bars display mean \pm s.e.m. (non-COVID-19: all $n=3$ patients, COVID-19: cluster $24 ; n=6$, cluster 27; $n=4$, all other clusters $n=7, p$-value was determined by two-sided $\mathrm{t}$-test). (h) Median expression level of indicated genes in TGFB1 ${ }^{+}$SARS-CoV-2negative cells and $T G F B 1^{+}$SARS-CoV-2-positive cells of all COVID-19 samples. Significance was calculated using the two-sided Mann-Whitney $U$-test. 
a

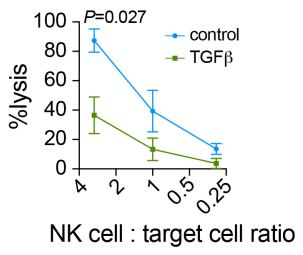

e

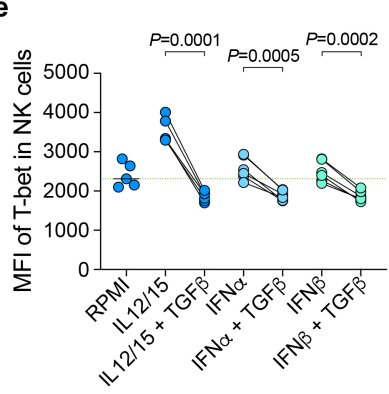

i

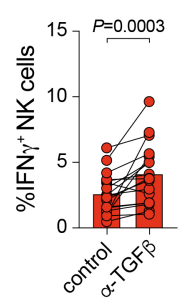

o

n

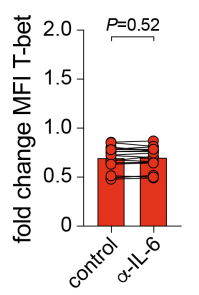

\section{b}

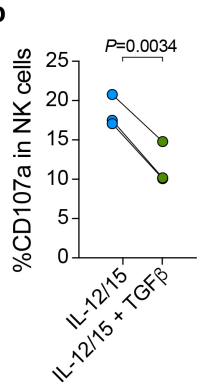

C

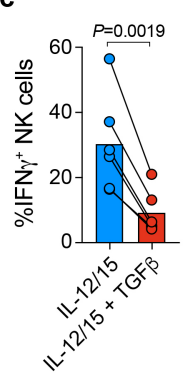

d

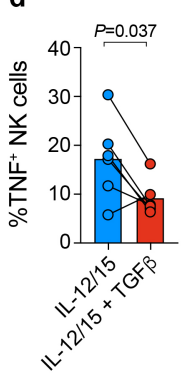

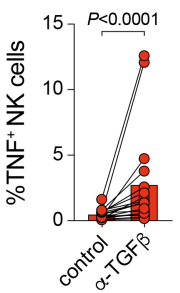

f

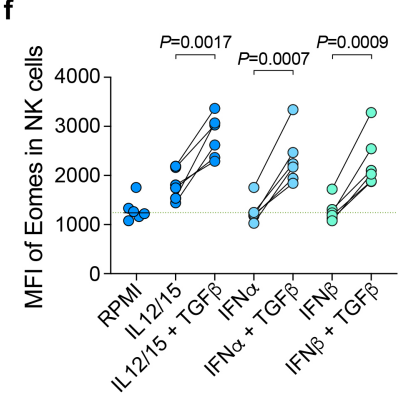

j

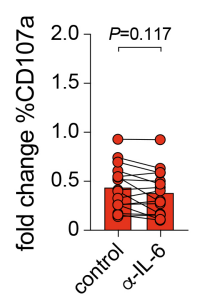

p

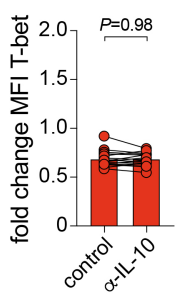

k

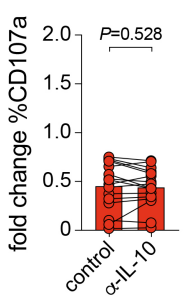

g

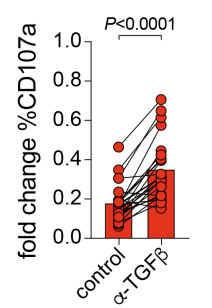

I

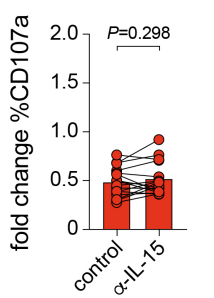

h

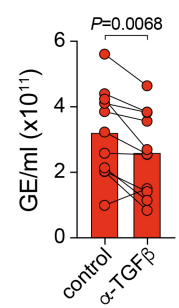

m

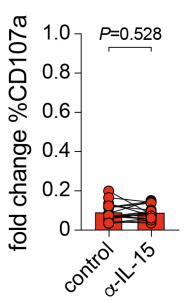

Extended Data Fig. 10 | Serum from severe COVID-19 patients suppresses NK cells in a TGF $\beta$-dependent manner. (a) NK cells were cultured in medium with (green) or without TGF $\beta$ (blue). Specific lysis (mean \pm s.e.m.; $n=3$ healthy donors) of $\mathrm{K} 562$ target cells was determined in a ${ }^{51} \mathrm{Chromium}$ release assay. Significance determined by two-sided unpaired t-test. (b) Sorted NK cells of healthy donors were cultured in medium containing the indicated cytokines with and without TGF $\beta$. The frequency of $\mathrm{CD} 107 \mathrm{a}^{+} \mathrm{NK}$ cells was analyzed after 4 h co-culture with $K 562$ cells ( $n=3$ per group). (c,d) PBMC of healthy donors $(n=6)$ were cultured for 4 days with the indicated cytokines and the frequency (mean) of IFN $\gamma^{+}$(c) and $\mathrm{TNF}^{+} \mathrm{NK}$ cells (d) was determined after PMA/ Iono stimulation. (e,f) Sorted NK cells of healthy donors were cultured for 4 days in medium containing the indicated cytokines. The MFI of T-bet (e) and Eomes (f) was measured by flow cytometry. The dashed line indicates median MFI of NK cells cultured in medium only (RPMI and IL-12/15 in e $\mathrm{n}=5$ donors, all others $n=6)$. $(\mathbf{g}-\mathbf{p})$ Sorted NK cells from 3 to 4 healthy donors per experiment were cultured in medium containing the cytokines IL-2 and IL-12 (g, m), IL-12 and IL-15 (h-k, n-o) or IL-2 (I,p) either alone or with serum from 3 to 6 patients with severe COVID-19 per experiment. In a second condition, patient sera were pre-incubated as indicated with anti-TGF $\beta$, anti-IL-6, anti-IL-10 or anti-IL-15 antibody before adding to the culture. The frequency of $\mathrm{CD} 107 \mathrm{a}^{+} \mathrm{NK}$ cells $(\mathbf{g}, \mathbf{j}-\mathbf{m})$, the frequency of IFN ${ }^{+} / \mathrm{TNF}^{+} \mathrm{NK}$ cells after $4 \mathrm{~h}$ co-culture with $\mathrm{K} 562$ cells (i), the viral load after co-culture with SARS-CoV-2-infected Vero E6 cells (h) and the MFI of T-bet (n-p) was determined. Fold change frequency or MFI was calculated between NK cells cultured in patients' sera (+/- prior anti-TGF $\beta$ treatment) and NK cells cultured in medium only. Each dot represents NK cells from one healthy donor cultured with severe COVID-19 serum (+/- prior antiTGF $\beta$ treatment $)(\mathbf{g} ; \mathbf{n}=\mathbf{2 4}, \mathbf{i} ; \mathbf{n}=\mathbf{1 8}, \mathbf{j}-\mathbf{p} ; \mathbf{n}=\mathbf{1 6}, \mathbf{h} ; \mathbf{n}=\mathbf{1 1}$ pooled samples derived from 3 patients, NK cell:target cell ratio 1:3,1:1, 3:1, 9:1, bars represent mean). Statistical analysis was performed using two-sided paired t-test (b-f) or two-sided Wilcoxon matched-pairs rank test (g-p). 


\section{Reporting Summary}

Nature Research wishes to improve the reproducibility of the work that we publish. This form provides structure for consistency and transparency in reporting. For further information on Nature Research policies, see our Editorial Policies and the Editorial Policy Checklist.

\section{Statistics}

For all statistical analyses, confirm that the following items are present in the figure legend, table legend, main text, or Methods section.

n/a Confirmed

$\bigotimes$ The exact sample size $(n)$ for each experimental group/condition, given as a discrete number and unit of measurement

$\searrow$ A statement on whether measurements were taken from distinct samples or whether the same sample was measured repeatedly

The statistical test(s) used AND whether they are one- or two-sided

Only common tests should be described solely by name; describe more complex techniques in the Methods section.

Х $\square$ A description of all covariates tested

\A description of any assumptions or corrections, such as tests of normality and adjustment for multiple comparisons

$\square$ A full description of the statistical parameters including central tendency (e.g. means) or other basic estimates (e.g. regression coefficient)

$\triangle$ AND variation (e.g. standard deviation) or associated estimates of uncertainty (e.g. confidence intervals)

$\triangle$ For null hypothesis testing, the test statistic (e.g. $F, t, r$ ) with confidence intervals, effect sizes, degrees of freedom and $P$ value noted

$\checkmark$ Give $P$ values as exact values whenever suitable.

Х $\square$ For Bayesian analysis, information on the choice of priors and Markov chain Monte Carlo settings

Х $\square$ For hierarchical and complex designs, identification of the appropriate level for tests and full reporting of outcomes

$\square$ Estimates of effect sizes (e.g. Cohen's $d$, Pearson's $r$ ), indicating how they were calculated

Our web collection on statistics for biologists contains articles on many of the points above.

\section{Software and code}

Policy information about availability of computer code

Data collection Cell sorting for single cell sequencing was performed using a MA900 Multi-Application Cell Sorter (Sony Biotechnology). For in vitro cultures NK cells were sorted using a FACS Aria II Cell Sorter (BD Biosciences). Flow cytometry data was acquired using FACS Fortessa X20 (BD Biosciences).

Data analysis

Flow cytometry data was analyzed using FlowJo Software V10.3 (Treestar). Statistical analysis was performed using GraphPad - Prism (version 8).

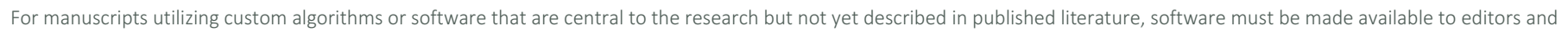

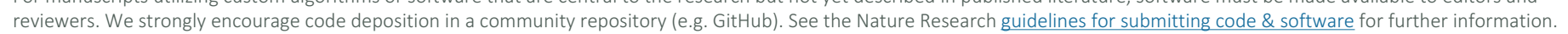

\section{Data}

Policy information about availability of data

All manuscripts must include a data availability statement. This statement should provide the following information, where applicable:

- Accession codes, unique identifiers, or web links for publicly available datasets

- A list of figures that have associated raw data

- A description of any restrictions on data availability

All generated sequencing data of this study were deposited into the NCBI Gene Expression Omnibus (accession number GSE184329) 


\title{
Field-specific reporting
}

Please select the one below that is the best fit for your research. If you are not sure, read the appropriate sections before making your selection.

\Life sciences

Behavioural \& social sciences

Ecological, evolutionary \& environmental sciences

For a reference copy of the document with all sections, see nature.com/documents/nr-reporting-summary-flat.pdf

\section{Life sciences study design}

All studies must disclose on these points even when the disclosure is negative.

\begin{tabular}{|c|c|}
\hline Sample size & Sample size was determined by the availability of patient material at the time of study \\
\hline Data exclusions & For selected analyses samples from patients receiving corticosteroids were excluded as indicated. \\
\hline Replication & $\begin{array}{l}\text { Each biological sample was analyzed independently (group sizes as indicated). For chromium release assays triplicates were used for each } \\
\text { biological sample. }\end{array}$ \\
\hline Randomization & $\begin{array}{l}\text { Not applicable as each patient sample (healthy controls, Flu-like illness, ambulant COVID-19/moderate COVID-19/severe COVID-19) was } \\
\text { analyzed indepently upon collection according to sample availability at the time of analysis. }\end{array}$ \\
\hline Blinding & $\begin{array}{l}\text { Blinding was not possible due to the nature of the samples analyzed. Patient samples (healthy controls, Flu-like illness, ambulant COVID-19, } \\
\text { moderate COVID-19, severe COVID-19) were obtained from different health care areas (outpatient clinic, regular ward, intensive care unit, } \\
\text { healthy controls also include health care workers). }\end{array}$ \\
\hline
\end{tabular}

\section{Reporting for specific materials, systems and methods}

We require information from authors about some types of materials, experimental systems and methods used in many studies. Here, indicate whether each material, system or method listed is relevant to your study. If you are not sure if a list item applies to your research, read the appropriate section before selecting a response.

\begin{tabular}{l|l}
\multicolumn{3}{c}{ Materials \& experimental syste } \\
\hline $\mathrm{n} / \mathrm{a}$ & Involved in the study \\
\hline & $\bigotimes$ Antibodies \\
$\square$ & $\square$ Eukaryotic cell lines \\
$\square$ & $\square$ Animals and other organisms \\
$\square$ & $\square$ Human research participants \\
$\square$ & $\square$ Clinical data
\end{tabular}

Antibodies

Antibodies used

\author{
Anti-human antibodies: \\ Flow Cytometry: \\ CD3 (BW264/56, VioBlue, Miltenyi Biotec, Cat No 130-113-133) \\ CD14 (TÜK4, VioBlue, Miltenyi Biotec, Cat No 130-113-152) \\ CD19 (LT19, VioBlue, Miltenyi Biotec, Cat No 130-113-172) \\ CD57 (TBO1, eF450, ThermoFisher, Cat No 48-0577-42) \\ Cell Proliferation Dye (VioBlue, ThermoFisher, Cat No 65-0842-85) \\ CD127 (A019D5, BV421, BioLegend, Cat No 351309) \\ NKp30 (p30-15, BV421, BD Bioscience, Cat No 563385) \\ Zombie Aqua Fixable Viability Kit (V500, BioLegend, Cat No 423101) \\ T-bet (4B10, BV605, BioLegend, Cat No 644817) \\ CD161 (HP-3G10, BV785, BioLegend, Cat No 339930) \\ Ki-67 (B56, PerCP-Cy5.5, BD Biosciences, Cat No 561284) \\ CD226 (11A8, PerCP-Cy5.5, BioLegend, Cat No 338314) \\ CD45 (QA17A19, PerCP/Cyanine5.5, BioLegend, Cat No 393409) \\ Granzyme B (GB11, PE, ThermoFisher, Cat No 12-8899-41) \\ TNF (Mab11, PE, BioLegend, Cat No 502908) \\ CD45 (HI30, PE, BioLegend, Cat No 304008) \\ CD117 (104D2, PE, Biolegend, Cat No 983304) \\ CD7 (CD7-6B7, PE/Dazzle, BioLegend, Cat No 343119)
}


CD69 (FN50, PE-Cy7, BD Bioscience, Cat No 557745)

IFNY (4S.B3, PE-Cy7, BioLegend, Cat No 502527)

NKp44 (2.29, PE-Cy7, Miltenyi Biotec, Cat No 130-120-487)

Eomes (WD1928, PE-Cy7, ThermoFisher, Cat No 25-4877-42)

Perforin (dG9, APC, ThermoFisher, Cat No 17-9994-42)

CD107a (H4A3, APC, BD Bioscience, Cat No 562622)

CD56 (AF12-7H3, APC, Miltenyi Biotec, Cat No 130-113-305)

CD8 (SFCI21, APC, Beckman Coulter, Cat No IM2469)

CD294 (BM16, APC, BioLegend, Cat No 350103)

NKp46 (9E2, APC, Miltenyi Biotec, Cat No 130-092-609)

CellTrace (APC, ThermoFisher, Cat No C34564)

CD56 (5.1H11, APC/Cyanine7, BioLegend, Cat No 362511)

CD3 (UCH-T1, APC-750, Beckman Coulter, Cat No A94680)

CD16 (3G8, BUV395, BD Bioscience, Cat No 563785)

CD3 (HIT3a, FITC, ThermoFisher, Cat No 11-0039-42)

CD4 (OKT4, FITC, ThermoFisher, Cat No 11-0048-42)

CD14 (HCD14, FITC, BioLegend, Cat No 325604)

CD19 (SJ25C1, FITC, BioLegend, Cat No 363008)

CD107a (eBioA4H3, FITC, eBioscience, Cat No 11-1079-42)

CD56 (N901, ECD, Beckman Coulter, Cat No A82943)

CD45 (J33, KrO, Beckman Coulter, Cat No B36294)

Validation

All purchased antibodies were validated by their manufacturers and further in-house testing.

- Miltenyi Biotec, https://www.miltenyibiotec.com/DE-en/lp/antibody-validation-improved-reproducibility.html

Three pillars of antibody validation: 1. Antibody reproducibility and consistency (Pure antibody products \& Lot-to-lot consistent performance); 2. Antibody specificity (Epitope competition assay, Knockout validation via targeted genome editing \& RNAi knockdown); 3. Antibody sensitivity (Functional testing of every product prior to release, Performance comparison \& Compatibility with fixation).

- BioLegend, https://www.biolegend.com/en-us/reproducibility

The reproducibility of published research has emerged as an urgent topic in today's scientific community. From funding agencies to researchers to manufacturers and publishers, it is critical for all of these groups to align themselves to ensure that research is done with rigor and is reproducible. How has BioLegend stepped up to meet these needs? In addition to sponsoring and collaborating with the Global Biological Standards Institute (GBSI) on setting antibody standards, we undertake extensive measures to ensure quality products that meet reproducibility requirements today and into the future.

BioLegend spends a considerable amount of effort in creating new antibodies for research. The majority of these new antibody products are monoclonal antibodies (mAb) produced from hybridomas. Clones of these hybridomas are carefully selected based on a number of criteria including robust growth and efficient production of a single clone of antibody that is specific to the intended target. The best clones move on to applications testing. All newly developed clones at BioLegend undergo validation testing for multiple applications. This serves as a cross-check for specificity and provides clarity for research uses. Maintaining lot-to-lot consistency is vital for reproducibility. It simply is not enough for antibody manufacturers to validate antibodies just once. Pass/fail specification requirements are essential for quality control testing of every lot of product. BioLegend maintains records for all lotspecific testing

- BD Biosciences, https://www.biocompare.com/Antibody-Manufacturing/355107-Antibody-Manufacturing-PerspectivesBDBioscience/

We conduct quality control (QC) testing in primary model systems to ensure biological accuracy in an ISO 9001 certified facility. BD carefully selects and characterizes antibody content in product development and tests in relevant primary model systems to ensure biological accuracy. BD conducts rigorous QC testing of each antibody lot tested side-by-side with a previously produced lot as reference. Our product development process includes testing on a combination of primary cells, cell lines and/or transfectant cell models with relevant controls using multiple immunoassays to ensure biological accuracy. We also perform multiplexing with additional antibodies to interrogate antibody staining in multiple cell populations. BD believes antibody validation is critical to ensure accurate scientific results. Both the consumer and the reagent provider share the responsibility for reproducible science.

- Invitrogen, https://www.thermofisher.com/de/de/home/life-science/antibodies/invitrogen-antibody-validation.html Antibodies are some of the most critical research reagents used in the lab. Poor specificity or application performance can significantly frustrate the ability to obtain good results, which can cause critical delays. Underperforming antibodies result in a lack of reproducibility, wasting time and money. In other words, researchers need antibodies that bind to the right target and work in their applications every time. To help ensure superior antibody results, we've expanded our specificity testing methodology using a 2-part approach for advanced verification. Part 1-Target specificity verification. This helps ensure the antibody will bind to the correct target. Our antibodies are being tested using at least 1 of the following methods to ensure proper functionality in researcher's experiments. Part 2-Functional application validation. These tests help ensure the antibody works in a particular application(s) of interest.

- Beckman Coulter, https://www.beckman.de/reagents/coulter-flow-cytometry/antibodies-and-kits/single-color-antibodies/qualitystandards

Development and production of our conjugated antibodies under current Good Manufacturing Practices (cGMP) in facilities that adhere and are certified to the highest standards in the industry.

Having more than 30 years of experience in conjugated antibody development and manufacturing, associated with the strictest internal quality controls that demonstrate lot-to-lot consistency over time in all our products.

\section{Eukaryotic cell lines}

Policy information about cell lines

Cell line source(s)

K562 (ATCC-10), Vero E6 (ATCC ${ }^{\circledR}$ CRL-1586 ${ }^{\text {TM }}$ ), Calu-3 (ATCC ${ }^{\circledR}$ HTB-55 $5^{\text {TM }}$ ) 
Mycoplasma contamination

Commonly misidentified lines (See ICLAC register)
Cells were not tested for mycoplasma contamination during the time of the study. Cell line viability $\geq 90 \%$ was confirmed prior co-culture.

\section{Human research participants}

Policy information about studies involving human research participants

Population characteristics

Recruitment

Ethics oversight
45 ambulant patients with COVID-19 (WHO 1 and 2 according to the WHO clinical ordinal scale) and 21 hospitalized patients with moderate COVID-19 (WHO3-4), 79 hospitalized patients with severe COVID-19 requiring ventilation (WHO 5-7) were enrolled in this study. All COVID-19 patients were tested positive for SARS-CoV-2

RNA via nasopharyngeal swabs. 20 patients presenting flu-like symptoms but were tested negative for SARS-CoV-2 (Flu-like illness, FLI) and a total of 96 healthy donors who did not present any clinical sign of viral infection were enrolled as controls.

All hospitalized patients were recruited from the regular ward or the Intensive Care Unit of the Charité. SARS-CoV-2 infection was verified by SARS-CoV-2 RNA via nasopharyngeal swabs. Ambulant COVID-19 patients (SARS-CoV-2 infection verified by SARS-CoV-2 RNA via nasopharyngeal swabs) and patients with Flu-like illness (negative for SARS-CoV-2

RNA via nasopharyngeal swabs) were recruited from the outpatient clinics in Berlin.

96 healthy donors who did not present any clinical sign of viral infection were enrolled as controls, recruitment in the Charité (health care workers) and in outpatient clinics.

Institutional Review Board of the Charité (EA2/066/20, EA2/072/20, EA4/014/20 and EA2/092/20)

\section{Flow Cytometry}

Plots

Confirm that:

Х The axis labels state the marker and fluorochrome used (e.g. CD4-FITC).

Х The axis scales are clearly visible. Include numbers along axes only for bottom left plot of group (a 'group' is an analysis of identical markers).

All plots are contour plots with outliers or pseudocolor plots.

Х A numerical value for number of cells or percentage (with statistics) is provided.

\section{Methodology}

Sample preparation

Instrument

Software

Cell population abundance

Gating strategy

Tick this box to confirm that a figure exemplifying the gating strategy is provided in the Supplementary Information.
Peripheral blood mononuclear cells (PBMC) were separated from peripheral blood by Pancoll human (PAN-Biotech) density gradient centrifugation at room temperature (RT). Cells were either used directly for analysis or stored in heat-inactivated fetal bovine serum (FCS, Pan- Biotech Cat\# P30-3602) with $10 \%$ DMSO at $-80^{\circ} \mathrm{C}$ prior to analysis. PBMC were incubated with Fc Blocking Reagent (Miltenyi Biotec) according to manufacturer's instructions. To exclude dead cells, the cells were stained with a LIVE/DEAD (LD) Fixable Aqua Dead Cell staining Kit (ThermoFisher, Cat\# L34965). For surface antigen staining the cells were incubated with monoclonal anti-human antibodies for $20 \mathrm{~min}$ at $4^{\circ} \mathrm{C}$. The Foxp3 Transcription Factor Staining Buffer Set (eBioscience, Cat\# 00-5523-00) was applied prior to intracellular staining of transcription factors, cytotoxic molecules and cytokines.

The samples were analyzed using FACS Fortessa X20 (BD Biosciences) for flow cytometry analysis. Cell sorting for single cell sequencing was performed using a MA900 Multi-Application Cell Sorter (Sony Biotechnology). For in vitro cultures NK cells were sorted using a FACS Aria II Cell Sorter (BD Biosciences).

Data was analyzed using FlowJo Software V10.3 (Treestar).

Cell population abundance was highly variable among patient groups and healthy controls. Purity of the sorted cell population was analyzed in a post-sort reanalysis. Cell counting for single cell sequencing was performed using a MACSQuant flow cytometer (Miltenyi Biotec).

NK cells were sorted as DAPI- CD3- CD14- CD19- CD45+ CD56+ (for sequencing) or CD3- CD14- CD19- CD4- CD45+ CD7+ CD56

+ (for in vitro experiments. Gating strategy for individual phenotyping experiments as indicated in the manuscript. 